\title{
STABLE SPLITTINGS OF THE DUAL SPECTRUM OF THE CLASSIFYING SPACE OF A COMPACT LIE GROUP
}

\author{
CHUN-NIP LEE
}

\begin{abstract}
For a compact Lie group $G$, there is a map from the $G$-equivariant fixed point spectrum of the zero sphere to the dual spectrum of the classifying space of $G, D B G_{+}$. When $G$ is finite, the affirmative solution to Segal's conjecture states that this map is an equivalence upon appropriate completion of the source. In the case of a compact Lie group, we obtain splitting results of $D B G_{+}$via this map upon taking $p$-adic completions.
\end{abstract}

\section{INTRODUCTION}

Let $G$ be a finite group, $A(G)$ be the Burnside ring of virtual finite $G$-sets, and $I(G)$ be the augmentation ideal of $A(G)$. Recall $E G$ is a contractible $G$-CW complex acting freely by $G$ whose quotient $E G / G$ is the classifying space of $G$, denoted by $B G$. Let ()$_{+}$denote the disjoint union with a base point. G. Segal in 1970 proposed the following

Conjecture. The map of G-equivariant stable cohomotopy groups induced by the projection $E G_{+} \rightarrow S^{0}$,

$$
\pi_{G}^{*}\left(S^{0}\right) \widehat{I(G)} \rightarrow \pi_{G}^{*}\left(E G_{+}\right) \cong \pi^{*}\left(B G_{+}\right),
$$

is an isomorphism, where ( $\hat{I}_{(G)}$ denotes the $I(G)$-adic completion.

In 1982, G. Carlsson [4] completed the proof of Segal's conjecture for all finite groups which had been studied previously with the works of Lin, Adams, Gunawardena, Miller, and May, among others. For more historical background information, see [4].

As for compact Lie groups, Segal's conjecture can also be formulated as above with an appropriate modification of the Burnside ring. However, it is known that in this case the conjecture does not hold in general. Since $\pi_{G}^{*}\left(S^{0}\right)$ is actually computable, we may interpret the Segal conjecture for finite groups as a statement about $\pi^{*}\left(B G_{+}\right)$. From this point of view, we could ask to what extent information about $\pi^{*}\left(B G_{+}\right)$is captured by $\pi_{G}^{*}\left(S^{0}\right)$ when $G$ is a compact Lie group.

We shall state our results in terms of spectra. For general references, see Adams [1] and Lewis-May-Steinberger [9]. Now suppose $G$ is a compact Lie

Received by the editors July 21, 1989 and, in revised form, January 11, 1990.

1980 Mathematics Subject Classification (1985 Revision). Primary 55P42; Secondary 55Q55.

The author was partially supported by the Alfred P. Sloan Dissertation Fellowship. 
group. The projection $E G_{+} \rightarrow S^{0}$ induces a map of spectra

$$
\Phi:\left(S^{0}\right)^{G}=F\left(S^{0}, S^{0}\right)^{G} \rightarrow F\left(E G_{+}, S^{0}\right)^{G} \simeq F\left(G B_{+}, S^{0}\right)=D B G_{+},
$$

where $\left(S^{0}\right)^{G}$ denotes the equivariant fixed point spectrum of the sphere $G$ spectrum and $F(, \quad)$ is the function space spectrum. By the results of tom Dieck [5, Theorem 2] and Lewis-May-Steinberger [9, Theorem 11.1], we have the following description:

$$
\left(S^{0}\right)^{G} \simeq \bigvee_{(H)} B W_{H}^{\mathrm{Ad} W_{H}},
$$

where $W_{H}$ is $N H / H, B W_{H}$ Ad $W_{H}$ is the Thom spectrum of the adjoint representation of $W_{H}$, and $(H)$ ranges over all conjugacy classes of closed subgroups of $G$. Notice that the summand corresponding to the trivial group is $B G^{\operatorname{Ad} G}$. When $G$ is finite, $B G^{\mathrm{Ad} G}=B G_{+}$and the affirmative solution to the Segal conjecture implies that $B G_{+} \hat{p}$ is a summand of $D B G_{+} \hat{p}$ given by the map $\Phi$ where we completed the spectra at a prime $p$. When $G$ is a compact Lie group, following evidence provided by Nishida [13], Ravenel [14], and Miller and Wilkerson [12], we prove the following:

Theorem 1.1. $B G^{\operatorname{Ad} G \hat{p}}$ is a summand of $D B G_{+} \hat{p}$ under $\Phi$.

In fact, we can extend this theorem to other conjugacy classes of finite $p$ subgroups of $G$. Specifically, we have

Theorem 3.1. Let $G$ be a compact Lie group and $p$ be a fixed prime. Suppose $H$ is a finite p-subgroup of $G$, then $B W_{H}{ }^{\mathrm{Ad} W_{H}} \hat{p}$ is a summand of $D B G_{+} \hat{p}$ under $\Phi$. Furthermore, the finite wedge sum of any such spectra splits from $D B G_{+} \hat{p}$.

There are technical difficulties in trying to extend Theorem 3.1 to conjugacy classes of closed infinite subgroups of $G$. In particular, the homotopy inverse limit of split cofiber sequences does not necessarily split. Nevertheless, we find the following weaker result to be true. Recall that a Lie group is said to be $p$-toral if it is an extension of a torus by a $p$-group.

Theorem 3.12. Let $G$ be a compact Lie group and $p$ be a fixed prime. Then the following map is an injection:

$$
\pi_{*}(\Phi): \bigoplus_{\substack{(H) \subset G \\ H \text { p-toral }}} \pi_{*}\left(B W_{H}^{\mathrm{Ad} W_{H}} \hat{p}\right) \rightarrow \pi_{*}\left(D B G_{+\hat{p}}\right) .
$$

Segal's conjecture for a general compact Lie group was first considered by $\mathbf{M}$. Feshbach in [7]. There he analyzed $\pi^{0}\left(B G_{+}\right)$and proved the conjecture via a different completion on $\pi_{G}^{0}\left(S^{0}\right)$ together with some additional assumptions at the prime 2 . Consequently, only those closed subgroups having finite index in their normalizers are being considered. What we accomplish is to give splitting results corresponding to finite subgroups which do not necessarily have finite index in their normalizers. Furthermore the corresponding injection result of Feshbach also holds for higher cohomotopy groups.

As an illustration of our techniques, combined with splitting results of Mitchell and Priddy [11], we obtain the following splitting for $G=S O(3)$ at the prime 2 . 
Theorem 4.13.

$$
\begin{aligned}
D B S O(3)_{+} \hat{2} \simeq & S_{2}^{0} \hat{2} \vee B S O(3)^{\mathrm{Ad} S O(3)} \hat{2} \\
& \vee \prod_{1}^{\infty} B O(2)^{\mathrm{Ad} O(2)} \hat{2} \vee \prod_{1}^{\infty} B Z_{2+\hat{2}} .
\end{aligned}
$$

The organization of this paper is as follows. In the first two sections, we shall concentrate on the proof of Theorem 1.1 in order to make the paper more readable. We first reduce to the case when $G$ is an extension of a torus by a $p$-group in $\S 1$. Using Segal's conjecture for $p$-groups, we express $D B G_{+} \hat{p}$ as a certain homotopy inverse limit. In $\S 2$, we analyze this homotopy inverse limit. The proof of Theorem 1.1 follows after a homotopy inverse limit of transfer maps is identified as a Thom spectrum. In the first part of $\S 3$, we show the method of the preceding two sections can be easily generalized to give Theorem 3.1. However, the proof of Theorem 3.12 is much more technical in nature, although the essential ideas are already contained in the first two sections. The new ingredient is the construction of a finite filtration of a system of spectra. The splitting result of $G=S O(3)$ at the prime 2 is the focus of $\S 4$. There we utilize the fact that we have an explicit description of all conjugacy classes of closed subgroups of $S O(3)$ together with the splitting results of [11]. Combined with a theorem of Miller and Wilkerson [12], this gives the splitting of $D B S^{3}+$ at the prime 2 .

Throughout this paper, we shall use $X^{G}$ to denote the $G$-equivariant fixed point spectrum of a $G$-spectrum $X$. Homotopy inverse limits will be denoted by $\lim _{\longleftarrow}$. (See Chapter XI of Bousfield-Kan [2] for details.) For a given nonequivariant spectrum $X$, the $p$-adic completion of $X$, denoted by $X_{\hat{p}}$, is $\lim _{i} X \wedge M\left(Z_{p^{i}}\right)$, where $M\left(Z_{p^{i}}\right)$ is the Moore spectrum of $Z_{p^{i}}$. Consult [14] for some of its basic properties.

The author wishes to thank his thesis advisor Professor Gunnar Carlsson for his constant encouragement and many suggestions without which this paper might not have been written.

\section{Preliminary Reductions}

Let $G$ be a compact Lie group. Denote by $G^{0}$ the connected component of the identity. When we say a maximal torus $T$ of $G$, we shall mean a maximal torus of $G^{0}$ in the usual sense. Consider the normalizer $N$ of $T$ in $G$. It is easy to see that $N / T$ is finite since $N / N \cap G^{0} \hookrightarrow G / G^{0}=\Gamma$ is finite. Furthermore, we have $G^{0} / N \cap G^{0} \cong G / N$. For if $\bar{g} \in G / N$, then $g T g^{-1}$, being a maximal torus of $G^{0}$, would be a conjugate of $T$ in $G^{0}$. Therefore, $\bar{g} \in G^{0} / N \cap G^{0}$. In particular, $\chi(G / N)=1$ since it is well known that $\chi\left(G^{0} / N \cap G^{0}\right)=1$.

Recall we want to prove

Theorem 1.1. $G B^{\operatorname{Ad} G} \hat{p}$ is a summand of $D B G_{+} \hat{p}$ under $\Phi$.

As a preliminary reduction, we have

Lemma 1.2. Let $N$ be the normalizer of a maximal torus $T$ of $G$. If $B N^{\operatorname{Ad} N_{\hat{p}}}$ is a summand of $D B N_{+} \hat{p}$ under $\Phi$, then Theorem 1.1, holds for $G$. 
Proof. We have the following diagram:

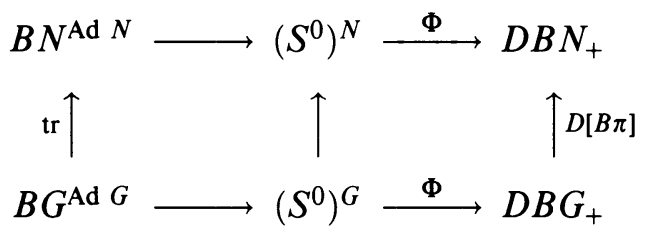

where $\pi$ is the inclusion $N \hookrightarrow G$ and $\operatorname{tr}$ is the associated bundle transfer (cf. Mann-Miller-Miller [10] and Nishida [13]). The diagram commutes by [13, Lemma 2.3] and the naturality of $\Phi$.

Consider the composite

$$
B G^{\mathrm{Ad} G} \stackrel{\operatorname{tr}}{\longrightarrow} B N^{\mathrm{Ad} N} \stackrel{j}{\longrightarrow} B N^{\mathrm{Ad} G} \stackrel{B \pi}{\longrightarrow} B G^{\mathrm{Ad} G},
$$

where $J$ is the inclusion $\operatorname{Ad} N \hookrightarrow \operatorname{Ad} G$.

Via Thom isomorphism, $\operatorname{tr}^{*}(\chi(\alpha) \cdot x)=\operatorname{tr}_{\circ}^{*}(x)$, where $\alpha=\operatorname{Ad} G / \operatorname{Ad} N$, $\chi(\alpha)$ is the Euler class of $\alpha, x \in H^{*}\left(B N, Z_{p}\right)$, and $\operatorname{tr}_{\circ}$ is the ordinary BeckerGottlieb transfer (cf. [13, Proposition 2.2]). Since $j^{*}$ is multiplication by $\chi(\alpha)$ and $\chi(G / N)=1$, it follows that the above composite induces an isomorphism on cohomology with $Z_{p}$ coefficient. Therefore $B G^{\operatorname{Ad} G \widehat{p}} \stackrel{\operatorname{tr}}{\longrightarrow} B N^{\operatorname{Ad} N \hat{p}}$ has a retraction. Hence by completing the above commutative diagram at a prime $p$, we have the desired result.

We are reduced to the case of the normalizer of a maximal torus of $G$. Suppose we have the extension

$$
1 \rightarrow T \rightarrow N \stackrel{\psi}{\rightarrow} W \rightarrow 1
$$

where $T$ is a torus and $W$ is a finite group.

Let $W_{p}$ be a $p$-Sylow subgroup of $W$ and $N_{p}$ the preimage of $W_{p}$ in $N$. We have

Lemma 1.3. If $B N_{p}{ }^{\mathrm{Ad}} N_{p} \hat{p}$ is a summand of $D B N_{p+\hat{p}}$, then $B N^{\operatorname{Ad} N_{\hat{p}}}$ is $a$ summand of $D B N_{+} \hat{p}$.

Proof. The proof is identical to that of Lemma 2.1 for $\chi\left(N / N_{p}\right) \not \equiv 0 \bmod p$.

Consequently, we may assume $W$ in $(*)$ to be a $p$-group. The following result by Feshbach is useful in analyzing $D B N_{+}$.

Proposition 1.4 [7, Theorem 1.1]. Let $|W|=n$, and $\widetilde{T}$ be the subgroup of $T$ generated by all elements of order $n$. Then there exists a subgroup $\widetilde{F}$ of $N$ such that $\widetilde{F} \cap T=\widetilde{T}$ and $\widetilde{F} / \widetilde{T} \cong W$. If $T^{\prime}$ is any invariant subgroup of $T$ containing $\widetilde{T}$, let $F^{\prime}$ denote the group generated by $T^{\prime}$ and $\widetilde{F}$. Then $F^{\prime} \cap T=T^{\prime}$ and $F^{\prime} / T^{\prime} \cong W$.

If $W$ is a $p$-group and $T_{Q_{p}}$ consists of all elements of order a power of $p$ in $T$, then we have

Corollary 1.5 [7, Corollary 1.2]. There exists a nested sequence $\left\{F_{i}\right\}$ of finite subgroups of $N$ such that $F_{i} / F_{i} \cap T \cong W$ with $\bigcup F_{i} \cap T=T_{Q_{p}}$. We denote $\bigcup F_{i}$ by $N_{Q_{p}}$. Furthermore, we can assume $F_{i} \cap T=\left\{x \in T \mid x^{p^{i}|W|}=1\right\}$.

Thus we have the following approximation result for $D B N_{+} \hat{p}$. 
Proposition 1.6. $D B N_{+\hat{p}}=\lim _{i} D B F_{i+\hat{p}}$.

Proof. It is easy to see that $H_{*}\left(B T_{Q_{p}}, Z_{p^{n}}\right) \stackrel{i_{*}}{\longrightarrow} H_{*}\left(B T, Z_{p^{n}}\right)$ is an isomorphism for $n \geq 1$, where $i: T_{Q_{p}} \rightarrow T$ is the inclusion. Consider the following map of extensions:

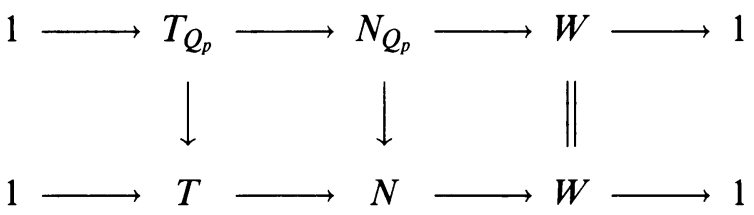

The homology with a $Z_{p^{n}}$ coefficient spectral sequence associated with the diagram induces an isomorphism of $E_{2}$ terms. Hence, $H_{*}\left(B N_{Q_{p}}, Z_{p^{n}}\right) \stackrel{\cong}{\longrightarrow}$ $H_{*}\left(B N, Z_{p^{n}}\right)$. Taking the direct limit of $n$ yields $H_{*}\left(B N_{Q_{p}}, Z_{p^{\infty}}\right) \stackrel{\cong}{\longrightarrow}$ $H_{*}\left(B N, Z_{p^{\infty}}\right)$. Now, since $Z_{p^{\infty}}=Z[1 / p] / Z$ and $\widetilde{H}_{*}\left(B N_{Q_{p}}, Z[1 / p]\right)=0$, we have $\widetilde{H}_{*}\left(B N_{Q_{p}}, Z\right) \cong \widetilde{H}_{*+1}\left(B N, Z_{p^{\infty}}\right)$ or $B N_{Q_{p}+} \rightarrow B N_{+} \wedge \Sigma^{-1} M\left(Z_{p^{\infty}}\right)$ is an equivalence, where $M\left(Z_{p^{\infty}}\right)$ denotes the Moore spectrum of $Z_{p^{\infty}}$.

Hence, $D B N_{Q_{p}+} \stackrel{\simeq}{\longleftarrow} D\left(B N_{+} \wedge \Sigma^{-1} M\left(Z_{p^{\infty}}\right)\right) \simeq D B N_{+\hat{p}}$ and therefore we have $\lim _{i} D B F_{i+\hat{p}}=D B N_{Q_{p}+\hat{p}} \simeq D B N_{+\hat{p}}$ as the desired result.

Since the $F_{i}$ are $p$-groups, the affirmative solution to Segal's conjecture for finite $p$-groups yields [4]:

$$
\pi:\left(S^{0}\right)^{F_{i}} \stackrel{\simeq}{\longrightarrow} D B F_{i+\hat{p}} .
$$

Furthermore, we have the commutative diagram:

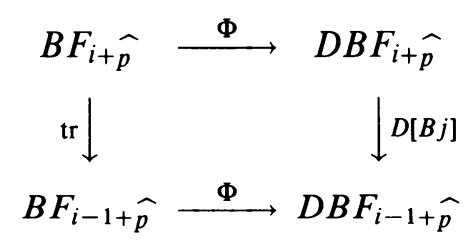

Here $j$ is the inclusion $F_{i-1} \hookrightarrow F_{i}$ and tr is the transfer associated to $j$. Even though $B F_{i+\hat{p}}$ is a summand of $D B F_{i+\hat{p}}$ under $\Phi$, it is not true that the splitting respects the maps in the inverse system $\left\{D B F_{i+\hat{p}}\right\}$. The method then is to find appropriate summands in $D B F_{i+\hat{p}}$ that will respect these maps in the inverse system. We begin by recalling the following

Definition. A family $\mathscr{F}$ in $G$ is a set of subgroups $G$ which is closed under conjugation and passage to subgroups.

Fix a closed subgroup $T^{\prime}$ of $T$. Consider the set

$$
\mathscr{I}_{T^{\prime}}=\left\{H \subset N \mid H \cap T \text { is a conjugate of } T^{\prime}\right\} .
$$

We have the following finiteness property of $\mathscr{I}_{T^{\prime}}$.

Lemma 1.7. There are a finite number of conjugacy classes in $\mathscr{I}_{T^{\prime}}$.

Proof. Recall we have the extension

$$
1 \rightarrow T \rightarrow N \rightarrow W \rightarrow 1 .
$$


Since $W$ is finite, it is sufficient to consider the subgroups in $\mathscr{I}_{T^{\prime}}$ whose image in $W$ is some fixed $J$. Suppose $H$ is such a subgroup. Let $N^{\prime}$ be the preimage of $J$ in $N$. Since $T^{\prime}$ is normal in $N^{\prime}$, we have the extension

$$
1 \rightarrow \frac{T}{T^{\prime}} \rightarrow \frac{N^{\prime}}{T^{\prime}} \rightarrow J \rightarrow 1
$$

Now $H$ corresponds to a splitting of (1.7.1). Two such splittings differ from a crossed homomorphism $J \rightarrow T / T^{\prime}$. Hence, it suffices to check $H^{1}\left(J, T / T^{\prime}\right)$ is finite. It is now clear that this reduces to showing $H^{1}\left(J,\left(S^{1}\right)^{n}\right)$ is finite, where $J$ acts on $\left(S^{1}\right)^{n}$ by automorphisms. Of course automorphisms of $\left(S^{1}\right)^{n}$ are induced from automorphisms of $R^{n}$, where $\left(S^{1}\right)^{n}=R^{n} / Z^{n}$. Thus we have an exact sequence of $Z[J]$-modules:

$$
0 \rightarrow Z^{n} \rightarrow R^{n} \rightarrow\left(S^{1}\right)^{n} \rightarrow 0 .
$$

Taking the corresponding long exact sequence yields

$$
\begin{aligned}
0 & \rightarrow H^{0}\left(J, Z^{n}\right) \rightarrow H^{0}\left(J, R^{n}\right) \rightarrow H^{0}\left(J,\left(S^{1}\right)^{n}\right) \rightarrow H^{1}\left(J, Z^{n}\right) \\
& \rightarrow H^{1}\left(J, R^{n}\right) \rightarrow H^{1}\left(J,\left(S^{1}\right)^{n}\right) \rightarrow H^{2}\left(J, Z^{n}\right) \rightarrow H^{2}\left(J, R^{n}\right) \rightarrow \cdots .
\end{aligned}
$$

Since $J$ is finite and $R^{n}$ is divisible with no elements of finite order, we have $H^{m}\left(J, R^{n}\right)=0$ for $m \geq 1$. However, $H^{2}\left(J, Z^{n}\right)$ is a finitely generated $Z$-module with $J$ finite. Therefore, $H^{2}\left(J, Z^{n}\right)$ is finite and so is $H^{1}\left(J,\left(S^{1}\right)^{n}\right)$.

Let $I_{m}=\left\{x \in T \mid x^{p^{m}}=1\right\}$. Consider the family $\mathscr{I}_{m}=\left\{H \subset N \mid H \cap T \subset I_{m}\right\}$. The following two results from [7] show that for each $H \in \mathscr{I}_{m}$, there exists a subgroup $H^{\prime} \in \mathscr{I}_{m}$ with $H^{\prime} \subset N_{Q_{p}}$ and $\left(H^{\prime}\right)=(H)$.

Proposition 1.8 [7, Theorem 1.4]. Suppose $W$ is a p-group. Let $H$ be a subgroup of $N$ such that $H \cap T=T_{Q_{p}}$ and $H / T_{Q_{p}} \cong W$. Then $H$ is conjugate to $N_{Q_{p}}$ in $N$.

Corollary 1.9 [7, Corollary 1.5]. Suppose $H$ is a subgroup of $N$ such that $H \cap$ $T \subset T_{Q_{p}}$. Then $H$ is conjugate to a subgroup of $N_{Q_{p}}$.

Now given a family $\mathscr{M}$ in $G$, there exists a universal $G$-space $E \mathscr{M}$ such that the $H$-fixed point of $E \mathscr{M}$ is empty if $H \notin \mathscr{M}$ and contractible otherwise (cf. tom Dieck [6, Proposition 7.2.1]).

Recall we have the family $\mathscr{J}_{n} . E \mathscr{I}_{n+}$ is an $N$-space. Upon restriction to $F_{i}, E \mathscr{I}_{n+}$ becomes an $F_{i}$-space.

Proposition 1.10. $\lim _{i}\left(E \mathscr{I}_{n+}\right)^{F_{i}}{ }_{p}$ is a summand of $D B N_{+} \hat{p}$.

Proof. Recall $D B N_{+\hat{p}}=\lim _{i} D B F_{i+\hat{p}}$. By Segal's conjecture for finite $p$ groups, $D B F_{i+\hat{p}}=\left(S^{0}\right)^{F_{i}} \hat{p}$. Consider the map $\left(E \mathscr{I}_{n+}\right)^{F_{i}} \stackrel{j}{\longrightarrow}\left(S^{0}\right)^{F_{i}}$ induced from $E \mathscr{I}_{n+} \rightarrow S^{0}$. By the naturality of the following splitting due to tom Dieck [5, Theorem 2],

$$
\pi_{*}^{G}(X) \cong \bigoplus_{(H)} \pi_{*}^{W_{H}}\left(E W_{H+} \wedge X^{H}\right)
$$

we see that

$$
j:\left(E \mathscr{J}_{n+}\right)^{F_{i}} \simeq \bigvee_{\substack{(H) \\ H \subset F_{i} \\ H \cap T \subset I_{n}}} B W_{H+}^{i} \rightarrow \bigvee_{\substack{(H) \\ H \subset F_{i}}} B W_{H+}^{i} \simeq\left(S^{0}\right)^{F_{i}}
$$


is the obvious map of inclusion, where $W_{H}^{i}=N_{i} H / H, N_{i} H$ is the normalizer of $H$ in $F_{i}$.

Suppose we identify each summand above by its corresponding conjugacy class $(H)$. Clearly $j$ has a splitting map which collapses all the summands whose corresponding conjugacy class $(H)$ are such that $H \cap T \not \subset I_{n}$ to the base point. We claim that this splitting map is compatible with $\left(S^{0}\right)^{F_{i}} \stackrel{\gamma}{\longrightarrow}\left(S^{0}\right)^{F_{i-1}}$ for all sufficiently large $i$, where $\gamma$ is induced from $F_{i-1} \hookrightarrow F_{i}$. The proposition then follows upon taking homotopy inverse limit.

We will show below that under $\gamma,(H)$ will map to summands of the form $\left\{\left(H^{\alpha} \cap F_{i-1}\right)\right\}$, where $\alpha \in F_{i}$ and $H^{\alpha}=\alpha H \alpha^{-1}$. Taking this for granted and using the fact that $I_{n}$ is normal, it is now easy to check that for all sufficiently large $i, H \cap T \not \subset I_{n}$ implies the same for $H^{\alpha} \cap F_{i-1}$.

We verify the claim made during the proof of Proposition 1.10.

Proposition 1.11. Let $K_{1} \subset K_{2}$ be the inclusion of finite groups. Suppose we identify each summand of $\left(S^{0}\right)^{K_{1}}$ (resp. $\left.\left(S^{0}\right)^{K_{2}}\right)$ by its corresponding conjugacy class $(H)$. Then under the map $\left(S^{0}\right)^{K_{2}} \stackrel{\gamma}{\longrightarrow}\left(S^{0}\right)^{K_{1}},(H)$ maps to $\left\{\left(H^{g_{i}} \cap K_{1}\right)\right\}$, where $\left\{g_{i}\right\}$ is a set of representatives for the double coset space $K_{1} \backslash K_{2} / N_{K_{2}} H$. Furthermore, the map is given by

$$
B W_{H+} \stackrel{C_{g_{i}}}{\longrightarrow} B\left(W_{H}\right)^{g_{i}}+\stackrel{\mathrm{tr}}{\longrightarrow} B \frac{(N H)^{g_{i}} \cap K_{1}}{H^{g_{i}} \cap K_{1}}+\stackrel{B_{i}}{\longrightarrow} B \frac{N_{1}\left(H^{g_{i}} \cap K_{1}\right)}{H^{g_{i}} \cap K_{1}}+,
$$

where $C_{g_{i}}$ is induced from conjugation and $N_{1}\left(H^{g_{i}} \cap K_{1}\right)$ denotes the normalizer of $H^{g_{i}} \cap K_{1}$ in $K_{1}$.

Proof. We first recall that tom Dieck's splitting theorem, mentioned in the proof of Proposition 1.10, is given by (when $G$ is finite)

$$
\begin{aligned}
\pi_{*}^{W_{H}}\left(E W_{H_{+}}\right) & \stackrel{\alpha}{\longrightarrow} \pi_{*}\left(B W_{H+}\right) \stackrel{j}{\longrightarrow} \pi_{*}^{G}\left(B W_{H+}\right) \\
& \stackrel{\tau}{\longrightarrow} \pi_{*}^{G}\left(G_{+} \wedge_{N H} E W_{H+}\right) \stackrel{p}{\rightarrow} \pi_{*}^{G}\left(S^{0}\right),
\end{aligned}
$$

where the first left arrow is Adam's isomorphism, $j$ is the induced map of $G \rightarrow$ $\{e\}, \tau$ is the $G$-equivariant transfer associated to $G_{+} \wedge_{N H} E W_{H+} \rightarrow B W_{H+}$, and $p$ is the evident projection map (cf. Lewis-May-McClure [8, Theorem 1]).

We have the following commutative diagram:

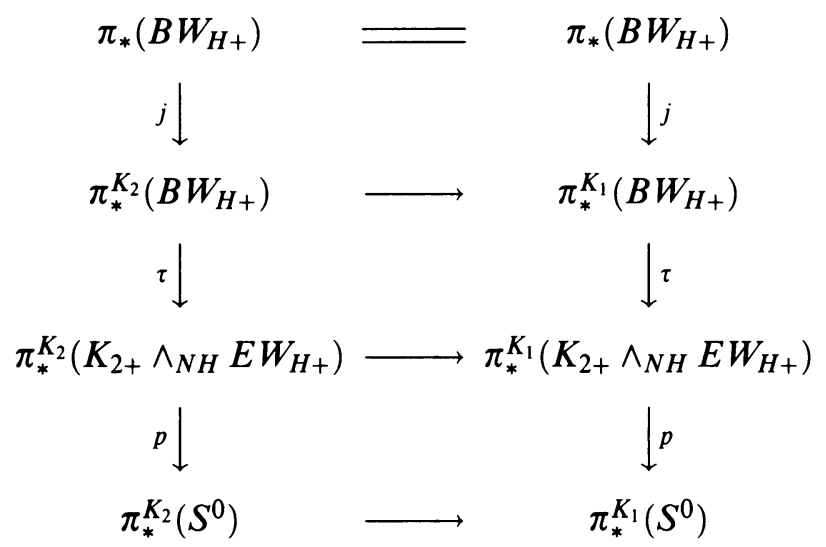

where the horizontal maps are induced from $K_{1} \hookrightarrow K_{2}$. 
Now we recall the construction of the $G$-equivariant transfer $\tau$. First there exists a $G$-map $\tilde{\tau}: S^{0} \rightarrow G / H_{+}$, called the pretransfer. Then taking the smash product with $E W_{H+}$ and the $W_{H}$ orbit gives $\tau$.

In the above diagram, we are interested in understanding the $K_{2}$-equivariant transfer as a $K_{1}$ map. Using the additivity property of transfers, we want to decompose $K_{2} / H$ as a $K_{1}$ space. The point is we have such a decomposition derived from that of $K_{2} / N H$. So suppose $\left\{g_{i}\right\}$ is a set of representatives of the double coset space $K_{1} \backslash K_{2} / N H$. Of course, by definition we have

$$
K_{2} / N H=\bigvee_{\left\{g_{i}\right\}} K_{1} /\left((N H)^{g_{i}} \cap K_{1}\right) .
$$

However, it is easy to see that as $K_{1}$ spaces, we also have

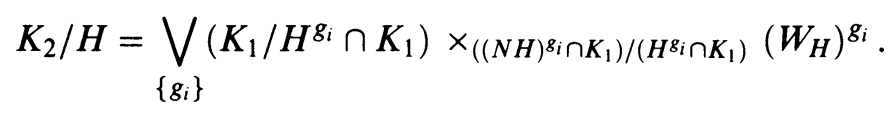

This observation gives rise to the following commutative diagram:

$$
\begin{array}{ccc}
\pi_{*}^{K_{1}}\left(B W_{H+}\right) & \pi_{*}^{K_{1}}\left(B W_{H+}\right) \\
\downarrow^{\tau} & \downarrow \oplus\left\{\theta_{g_{i}}\right\} \\
\pi_{*}^{K_{1}}\left(K_{2+} \wedge_{N H} E W_{H+}\right) \stackrel{\cong}{\longrightarrow} \bigoplus_{\left\{g_{i}\right\}} \pi_{*}^{K_{1}}\left(K_{1+} \wedge_{(N H)^{g_{i} \cap K_{1}}} E\left(W_{H}\right)^{g_{i}}\right)
\end{array}
$$

where $\theta_{g_{i}}$ is induced from appropriate equivariant transfers.

By using the commutativity property of transfers, we have the following diagram describing $\theta_{g_{i}}$ :

$$
\begin{gathered}
\pi_{*}^{K_{1}}\left(B W_{H+}\right) \stackrel{\theta_{g_{i}}}{\longrightarrow} \pi_{*}^{K_{1}}\left(K_{1+} \wedge(N H)^{g_{i} \cap K_{1}} E\left(W_{H}\right)^{g_{i}}\right) \\
\tau^{\prime} \circ C_{g_{i}} \downarrow \\
\tau_{*}^{K_{1}}(B \cong \\
\left.\frac{(N H)^{g_{i} \cap K_{1}}}{H^{g_{i} \cap K_{1}}+}\right) \stackrel{\tau}{\longrightarrow} \pi_{*}^{K_{1}}\left(K_{1+} \wedge_{(N H)^{g_{i} \cap K_{1}}} E \frac{(N H)^{g_{i} \cap K_{1}}}{H^{g_{i} \cap K_{1}}+}\right)
\end{gathered}
$$

Here $\tau^{\prime}$ is the evident transfer.

Combining the above commutative diagrams gives the following:

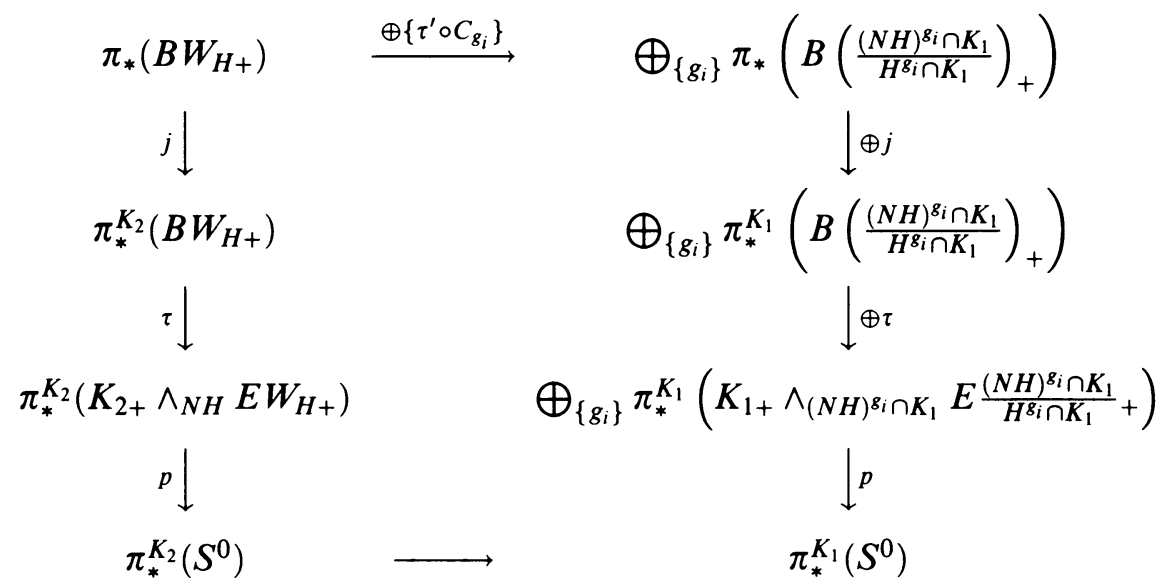


Now

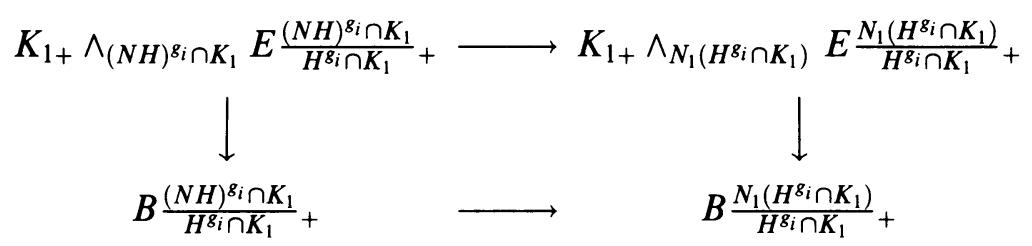

is a pullback diagram. So

$$
\begin{gathered}
\pi_{*}^{K_{1}}\left(B \frac{N_{1}\left(H^{g_{i} \cap K_{1}}\right)}{H^{g_{i} \cap K_{1}}}+\right) \stackrel{\tau}{\longrightarrow} \pi_{*}^{K_{1}}\left(K_{1+} \wedge_{N_{1}\left(H^{\left.g_{i} \cap K_{1}\right)} E \frac{N_{1}\left(H^{\left.g_{i} \cap K_{1}\right)}\right.}{H^{g_{i} \cap K_{1}}}+\right)}\right. \\
\uparrow \\
\pi_{*}^{K_{1}}\left(B \frac{(N H)^{g_{i} \cap K_{1}}}{H^{g_{i} \cap K_{1}}}+\right) \stackrel{\tau}{\longrightarrow} \pi_{*}^{K_{1}}\left(K_{1+} \wedge_{(N H)^{g_{i} \cap K_{1}}} E \frac{(N H)^{g_{i} \cap K_{1}}}{H^{g_{i} \cap K_{1}}+}\right)
\end{gathered}
$$

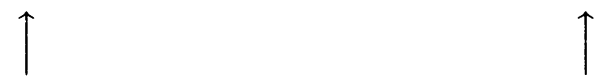

commutes. It is clear that we also have

$$
\begin{aligned}
& \pi_{*}^{K_{1}}\left(K_{1+} \wedge_{(N H)^{g_{i} \cap K_{1}}} E\left(W_{H}\right)^{g_{i}}\right) \longleftarrow \pi_{*}^{K_{1}}\left(K_{1+} \wedge_{(N H)^{g_{i} \cap K_{1}}} E \frac{(N H)^{g_{i} \cap K_{1}}}{H^{g_{i} \cap K_{1}}}+\right) \\
& p \downarrow \quad \pi_{*}^{K_{1}}\left(K_{1+} \wedge_{(N H)^{g_{i}} \cap K_{1}}\right. \\
& \pi_{*}^{K_{1}}\left(S^{0}\right) \quad \stackrel{p}{\longleftarrow} \pi_{*}^{K_{1}}\left(K_{1+} \wedge_{N_{1}\left(H^{g_{i}} \cap K_{1}\right)} E \frac{N_{1}\left(H^{g_{i}} \cap K_{1}\right)}{H^{g_{i} \cap K_{1}}}+\right)
\end{aligned}
$$

where all unmarked arrows are induced from evident inclusions.

Therefore, by combining these commutative diagrams, we see that the summand corresponding to $(H)$ goes to summands corresponding to $\left\{\left(H^{g_{i}} \cap K_{1}\right)\right\}$ with the prescribed maps as asserted.

For a general compact Lie group, tom Dieck's splitting theorem for $\pi_{*}^{G}\left(S^{0}\right)$ is given as follows:

$$
\begin{aligned}
& \pi_{*}^{W_{H}}\left(E W_{H+}\right) \stackrel{\cong}{\longrightarrow} \pi_{*}\left(B W_{H} \mathrm{Ad}^{W_{H}}\right) \rightarrow \pi_{*}^{G}\left(B W_{H}^{\mathrm{Ad} W_{H}}\right) \\
& \quad \stackrel{\tilde{\tau}}{\longrightarrow} \pi_{*}^{G}\left(G_{+} \wedge_{N H} B W_{H}^{\mathrm{Ad} W_{H}}\right) \stackrel{1 \wedge \tau}{\longrightarrow} \pi_{*}^{G}\left(G_{+} \wedge_{N H} E W_{H+}\right) \stackrel{p}{\longrightarrow} \pi_{*}^{G}\left(S^{0}\right),
\end{aligned}
$$

where $\tilde{\tau}$ is the pretransfer associated with $G / N H \rightarrow 1$ and $\tau$ is the dimensionshifting transfer associated with $W_{H} \rightarrow 1$ (cf. [9, Chapter IV]). The analogue of the previous proposition is the following

Proposition 1.12. Let $K_{1}, H \subset K_{2}$ be inclusions of compact Lie groups. Consider the double coset space $K_{1} \backslash K_{2} / N_{K_{2}} H$. Let $\left\{M_{\alpha}\right\}$ be the set of all orbit type manifold components of the double coset space. Choose $g_{\alpha} \in N$ for each $\alpha$ which projects into $M_{\alpha}$. Suppose we identify each summand of $\left(S^{0}\right)^{K_{1}}$ (resp. $\left(S^{0}\right)^{K_{2}}$ ) by its corresponding conjugacy class $(H)$. Then under the map $\left(S^{0}\right)^{K_{2}} \stackrel{\gamma}{\longrightarrow}\left(S^{0}\right)^{K_{1}},(H)$ maps to $\left\{\left(H^{g_{\alpha}} \cap K_{1}\right)\right\}$. Furthermore, each map is given by $\chi^{\#}\left(M_{\alpha}\right)$ times

$$
B W_{H}^{\text {Ad } W_{H}} \stackrel{C_{g_{\alpha}}}{\longrightarrow} B W_{H^{g_{\alpha}}} \text { Ad } W_{H{ }^{g}} \stackrel{\operatorname{tr}}{\longrightarrow} B W_{H_{1}} \text { Ad } W_{H_{1}} \stackrel{B i}{\longrightarrow} B W_{H_{2}}{ }^{\text {Ad } W_{H_{2}}},
$$


where $\chi^{\#}\left(M_{\alpha}\right)=\chi\left(\bar{M}_{\alpha}\right)-\chi\left(\bar{M}_{\alpha}-M_{\alpha}\right)$ is the internal Euler characteristic, $C_{g_{\alpha}}$ is induced from conjugation, $W_{H_{1}}=\left((N H)^{g_{\alpha}} \cap K_{1}\right) /\left(H^{g_{\alpha}} \cap K_{1}\right), \quad W_{H_{2}}=$ $\left(N_{1}\left(H^{g_{\alpha}} \cap K_{1}\right)\right) /\left(H^{g_{\alpha}} \cap K_{1}\right)$, and $N_{1}\left(H^{g_{\alpha}} \cap K_{1}\right)$ denotes the normalizer of $H^{g_{\alpha}} \cap K_{1}$ in $K_{1}$.

Remark. We will omit the proof since it proceeds similarly to that of Proposition 1.11. Note the internal Euler characteristic comes in when one decomposes a certain $K_{2}$-space as a $K_{1}$-space. See Theorem 2.11 of Chapter IV in [9] for more details.

\section{Proof of TheOREM 1.1}

Consider the map $\left(E \mathscr{I}_{n+}\right)^{N} \widehat{p} \stackrel{\gamma}{\longrightarrow} \lim _{i}\left(E \mathscr{I}_{n+}\right)^{F_{i}}$ induced from inclusions $F_{i} \hookrightarrow N$. Notice that we have

$$
\left(E \mathscr{I}_{n+1}\right)^{N} \hat{p}=\bigvee_{\substack{(H) \\ H \cap T \subset I_{n}}} B W_{H} \operatorname{Ad}_{W_{H}} \widehat{p} .
$$

Since $B N^{\operatorname{Ad} N} \stackrel{\Phi}{\longrightarrow} D B N_{+} \hat{p}$ is compatible with $\gamma$, it therefore follows from Proposition 1.10 that it suffices to show $\gamma$ is an equivalence. We might add that it would be enough to assume $n=0$ for proving Theorem 1.1. However, for later purposes, we will prove $\gamma$ is an equivalence for all $n$.

In order to analyze $\varliminf_{i}\left(E \mathscr{J}_{n+}\right)^{F_{i}} \hat{p}$, we use Lemma 1.7. Let $\mathscr{F}_{1} \subset \mathscr{F}_{2}$ be adjacent families of $N$ contained in $\mathscr{I}_{n}$, i.e., $\mathscr{F}_{2}-\mathscr{F}_{1}=(H)$. We have the following map of cofibrations:

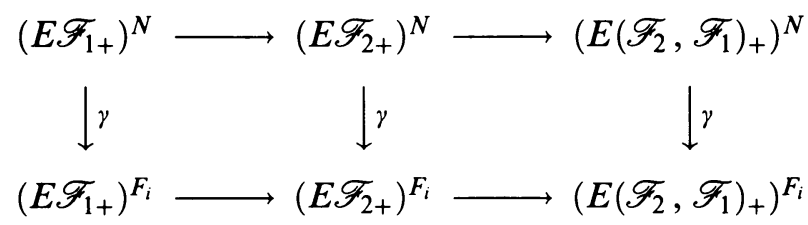

where the $K$-fixed point of $E\left(\mathscr{F}_{2}, \mathscr{F}_{1}\right)$ is $*$ if $(K) \neq(H)$ in $N$, or $S^{0}$ if $(K)=(H)$ in $N$. By completing the above map of cofibration sequences at the prime $p$ and taking the homotopy inverse limit, we have the following map of cofibration sequences:

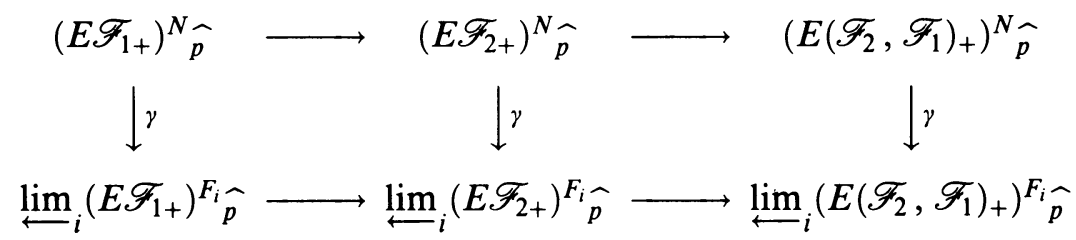

Hence, it suffices to prove

Proposition 2.1. Let $\mathscr{F}_{1} \subset \mathscr{F}_{2}$ be adjacent families of $\mathscr{I}_{n}$. Then we have the equivalence

$$
\left(E\left(\mathscr{F}_{2}, \mathscr{F}_{1}\right)_{+}\right)^{N} \hat{p} \underset{\gamma}{\simeq} \underbrace{}_{i} \lim _{i}\left(E\left(\mathscr{F}_{2}, \mathscr{F}_{1}\right)_{+}\right)^{F_{i}} \hat{p} .
$$

We need some preliminary results. Fix $(H)=\mathscr{F}_{2}-\mathscr{F}_{1}$. The map

$$
\left(E\left(\mathscr{F}_{2}, \mathscr{F}_{1}\right)_{+}\right)^{N} \hat{p} \stackrel{\gamma}{\longrightarrow}\left(E\left(\mathscr{F}_{2}, \mathscr{F}_{1}\right)_{+}\right)^{F_{i}} \hat{p}
$$


is the same as

$$
B W_{H} \text { Ad } W_{H} \hat{p} \rightarrow \bigvee_{\substack{\left(H^{\prime}\right) \\ H^{\prime} \subset F_{i} \\\left(H^{\prime}\right)=(H) \text { in } N}} B N_{i}\left(H^{\prime}\right) / H^{\prime}+\hat{p},
$$

where $N_{i}\left(H^{\prime}\right)$ denote the normalizer of $H^{\prime}$ in $F_{i}$. Notice the map in $(2.1 .1)$ is described by Proposition 1.12.

By Corollary 1.9, we may assume $H \subset F_{i_{\circ}}$ for some $i_{\circ}$. We give an explicit description of the $\left(H^{\prime}\right)$ 's in (2.1.1).

Lemma 2.2. The $N H$ orbit of $\left(F_{i} \backslash N\right)^{H}$ with action on the right is in one-to-one correspondence with $\left\{\left(H^{\prime}\right) \mid H^{\prime} \subset F_{i},\left(H^{\prime}\right)=(H)\right.$ in $\left.N\right\}$. The assignment is given by $\beta:\{\bar{g}\} \rightarrow\left(H^{g}\right)$, where $H \subset F_{i}$. In particular, the set $\{\bar{g}\}$ is finite.

Proof. Let $\bar{g} \in F_{i} \backslash N$ be fixed under $H$. Therefore we have $\bar{g} H=\bar{g}$ or $g \mathrm{Hg}^{-1} \subset F_{i}$. Furthermore, let $k \in N H$ and $z \in F_{i}$; then $H^{z g k}=$ $z g k H k^{-1} g^{-1} z^{-1}=z g H g^{-1} z^{-1} \subset z F_{i} z^{-1}=F_{i}$. Hence, $\beta$ is well defined since $\left(H^{z k k}\right)=\left(H^{g}\right)$ in $F_{i}$.

Suppose $\bar{g}_{1}, \bar{g}_{2}$ are two representatives such that $\left(H^{g_{1}}\right)=\left(H^{g_{2}}\right)$ in $F_{i}$. This implies $g_{2}^{-1} h g_{1} \in N H$ for some $h \in F_{i}$. Hence, $\bar{g}_{1}=\bar{g}_{2}\left(g_{2}^{-1} h g_{1}\right)$ or $\bar{g}_{1} \sim \bar{g}_{2}$ in the $N H$ orbit of $\left(F_{i} \backslash N\right)^{H}$. $\beta$ is injective.

Clearly if $\left(H^{\prime}\right)=(H)$ in $N$, then $H^{\prime}=H^{g}$ for some $g \in N$. Thus, $\beta(\bar{g})=\left(H^{g}\right)$ with $\bar{g}$ fixed under $H$. So $\beta$ is surjective and we are done.

Hence to each $i \geq i_{\circ}$, we have a set of representatives $\left\{\bar{g}_{\alpha}^{(i)}\right\}$ in the $N H$ orbit of $\left(F_{i} \backslash N\right)^{H}$ corresponding to summands of $\left(E\left(\mathscr{F}_{2}, \mathscr{F}_{1}\right)_{+}\right)^{F_{i}} \hat{p}$. From Proposition 1.11 , we get

Lemma 2.3. Let $j:\left(F_{i} \backslash N\right)^{H} / N H \rightarrow\left(F_{i+1} \backslash N\right)^{H} / N H$ be the induced map of $F_{i} \hookrightarrow F_{i+1}$. Suppose

$$
\left.j^{-1}\left(\bar{g}_{\alpha}^{(i+1)}\right)\right)=\left\{\bar{g}_{\alpha_{1}}^{(i)}, \bar{g}_{\alpha_{2}}^{(i)}, \ldots, \bar{g}_{\alpha_{m}}^{(i)}\right\} .
$$

Then the summand $\left(H^{g_{\alpha}^{(i+1)}}\right)$ in $\left(E\left(\mathscr{F}_{2}, \mathscr{F}_{1}\right)_{+}\right)^{F_{i+1}}$ maps to summands $\left(H^{g_{\alpha_{1}}^{(i)}}\right)$, $\left(H^{g_{\alpha_{2}}^{(i)}}\right), \ldots,\left(H^{g_{\alpha_{m}}^{(i)}}\right)$ in $\left(E\left(\mathscr{F}_{2}, \mathscr{F}_{1}\right)_{+}\right)^{F_{i}}$ under the inclusion $F_{i} \hookrightarrow F_{i+1}$.

Proof. Without loss of generality, we may assume $g_{\alpha}^{(i+1)}=g_{\alpha_{1}}^{(i)}=g$. Let $\left\{z_{\gamma}\right\}$ be the set of representatives of the double coset:

$$
F_{i} \backslash F_{i+1} /(N H)^{g} \cap F_{i+1} \text {. }
$$

Proposition 1.11 shows that $\left(H^{g}\right)$ maps to $\left\{\left(H^{z_{\gamma} g} \cap F_{i}\right)\right\}$ in $\left(S^{0}\right)^{F_{i+1}} \rightarrow\left(S^{0}\right)^{F_{i}}$. Hence, we only have to consider those $z_{\gamma}$ such that $H^{z_{\gamma} g} \subset F_{i}$. Now $j\left(\bar{z}_{\gamma} g\right)=$ $\bar{g}$ and hence $\bar{z}_{\gamma} g \in\left\{\bar{g}_{\alpha_{1}}^{(i)}, \ldots, \bar{g}_{\alpha_{m}}^{(i)}\right\}$.

Proposition 2.4.

$$
{\underset{i}{i}}_{\lim }\left(E\left(\mathscr{F}_{2}, \mathscr{F}_{1}\right)_{+}\right)^{F_{i}} \underset{p}{\stackrel{\cong}{\longrightarrow}} \underset{\lim }{\lim }\left(\frac{N H \cap F_{i}}{H}\right)_{+p} .
$$

Proof. From the previous lemma, it is obvious that only the summand corresponding to $(H)$ in $\left(E\left(\mathscr{F}_{2}, \mathscr{F}_{1}\right)_{+}\right)^{F_{i+1}}$ will map to the summand corresponding to $(H)$ in $\left(E\left(\mathscr{F}_{2}, \mathscr{F}_{1}\right)_{+}\right)^{F_{i}}$. By Proposition $1.11, B\left(\left(N H \cap F_{i+1}\right) / H\right)_{+}$maps 
to $B\left(\left(N H \cap F_{i}\right) / H\right)_{+}$by transfer. Hence we have the following map of cofiber sequences:

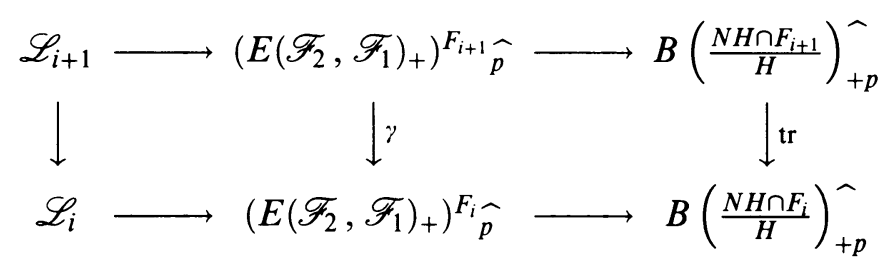

where $\mathscr{L}_{i+1}$ and $\mathscr{L}_{i}$ are the fibers of right horizontal maps.

Now Lemma 2.3 gives us a description of $\gamma$ in terms of the corresponding representatives $\left\{\bar{g}_{\alpha}^{(i)}\right\}$. If we can show for a fixed $i$ that there exists $d(i)$ so that we can choose the $g_{\alpha}^{(i)}$,s belonging to $F_{d(i)}$ for all $\alpha$, then we would be done. This is because $\mathscr{L}_{d(i)} \rightarrow \mathscr{L}_{i}$ would then be the trivial map. Upon taking the homotopy inverse limit this yields

$$
{\underset{\lim }{\operatorname{tr}}} B\left(\frac{N H \cap F_{i}}{H}\right)_{+p} \stackrel{\simeq}{\lim _{i}}\left(E\left(\mathscr{F}_{2}, \mathscr{F}_{1}\right)_{+}\right)^{F_{i}} \hat{p} .
$$

The claim follows from the following lemma.

Lemma 2.5. Let $\left\{\bar{g}_{\alpha}^{(i)}\right\}$ be representatives of $\left(F_{i} \backslash N\right)^{H} / N H$. There exists $d(i)$ such that $\left\{g_{\alpha}^{(i)}\right\}$ can be chosen so that $g_{\alpha}^{(i)} \in F_{d(i)}$ for all $\alpha$.

Proof. Since $\widetilde{F} \hookrightarrow F_{i}$, we can assume each $g_{\alpha}^{(i)} \in T$. For each $x \in H$, pick $\tilde{x} \in \widetilde{F}$ so that $\tilde{x}^{-1} x \in T$. Therefore $g_{\alpha}^{(i)} x g_{\alpha}^{(i)^{-1}}=\tilde{x}\left(\tilde{x}^{-1} x\right)\left(\tilde{x}^{-1} g_{\alpha}^{(i)} \tilde{x}\right) g_{\alpha}^{(i)^{-1}} \in F_{i}$ since $\tilde{x}^{-1} g_{\alpha}^{(i)} \tilde{x}, \tilde{x}^{-1} x \in T$. But elements of $F_{i} \cap T$ have order dividing $p^{m}$ for some fixed integer $m$. Therefore we have $\left(\tilde{x}^{-1} g_{\alpha}^{(i)} \tilde{x}\right)^{p^{m}}\left(g_{\alpha}^{(i)}\right)^{-p^{m}}=1$. Or equivalently, $x^{-1} g_{\alpha}^{(i) p^{m}} x g_{\alpha}^{(i)^{-p^{m}}}=1$. Hence, $g_{\alpha}^{(i)^{p^{m}}} \in N H \cap T$.

Next, we show $\left\{g_{\alpha}^{(i)}\right\}$ can be chosen to have finite order. Let $z=g_{\alpha}^{(i)}$. Then $z$ generates an abelian group $L$. Since the closure $\bar{L}$ is a compact abelian Lie group with a cyclic generator, it therefore has the form of $J \times T^{k}$ with $J$ cyclic. Similarly, $z^{p^{m}}$ generates $L^{\prime}$ with $\bar{L}^{\prime}=J^{\prime} \times T^{k^{\prime}}$. Since $y \in J \times T^{k} \Rightarrow$ $y^{p^{m}} \in J^{\prime} \times T^{k^{\prime}}$, it is clear that $\bar{L}^{\prime}=J^{\prime} \times T^{k}$ with $J^{\prime}$ a cyclic subgroup of $J$.

The above analysis shows that there exists $z^{\prime} \in \bar{L}^{\prime}$ such that $\left(z^{\prime}\right)^{-1} z$ is of finite order. Furthermore, $z^{p^{m}} \in N H \Rightarrow \bar{L}^{\prime} \subset N H$ and so $z^{\prime} \in N H$. Therefore we may assume $g_{\alpha}^{(i)}$ to be of finite order.

So we suppose $\left(g_{\alpha}^{(i)}\right)^{M}=1$. Write $g_{\alpha}^{(i)}=\left(g_{\alpha}^{(i)}\right)^{1-p^{l}}\left(g_{\alpha}^{(i)}\right)^{p^{l}}$. Also $\left(g_{\alpha}^{(i)}\right)^{p^{l}} \in$ $N H$ if $l \geq m$. We can pick $l \geq m$ so that $\left(g_{\alpha}^{(i)}\right)^{1-p^{l}}$ is of order a power of $p$. We have proved the lemma.

By Proposition 2.4, it suffices to know the map between

$$
B W_{H}^{\mathrm{Ad} W_{H} \hat{p}}=\left(E\left(\mathscr{F}_{2}, \mathscr{F}_{1}\right)_{+}\right)_{\hat{p}}^{N} \text { and } B\left(\frac{N H \cap F_{i}}{H}\right)_{+p} \text {. }
$$

From Proposition 1.12, this amounts to evaluating the internal Euler characteristics of the orbit type manifold component corresponding to 1 in $F_{i} \backslash N / N H$.

Proposition 2.6. Let $H \subset K_{1} \subset K_{2}$ be an inclusion of compact Lie groups. Denote by $\mathrm{NH}$ the normalizer of $\mathrm{H}$ in $K_{2}$. Let $M_{\circ}$ be the orbit type manifold 
component corresponding to 1 in $K_{1} \backslash K_{2} / N H$. Then $M_{\circ}$ is a point. In particular, $\chi^{\#}\left(M_{\circ}\right)=1$.

Proof. Suppose $z$ has the same orbit type as 1 . This would mean that $K_{1}^{z^{-1}} \cap$ $N H$ is a conjugate of $K_{1} \cap N H$ in $N H$. So $K_{1}^{\gamma z^{-1}} \cap N H=K_{1} \cap N H$ for some $\gamma \in N H$. However, since $H \subseteq K_{1} \cap N H$, this implies $H \subseteq K_{1}^{z^{-1}}$ or $H^{z} \subseteq K_{1}$. In other words, $z$ actually belongs to the $N H$ orbit of $\left(K_{1} \backslash K_{2}\right)^{H}$. But it was shown in Bredon [3] that $\left(K_{1} \backslash K_{2}\right)^{H} / N H$ is finite. Therefore we can conclude that $z$ is not in the same orbit type manifold component as 1 unless $z=1$. Therefore $M_{\circ}$ is a point.

The above discussion together with Proposition 1.12 implies that

$$
\left(E\left(\mathscr{F}_{2}, \mathscr{F}_{1}\right)_{+}\right)^{N} \hat{p} \rightarrow \underset{i}{\lim }\left(E\left(\mathscr{F}_{2}, \mathscr{F}_{1}\right)_{+}\right)^{F_{i}} \hat{p}
$$

is equivalent to

$$
B W_{H} \mathrm{Ad}_{H} \widehat{p} \stackrel{\operatorname{tr}}{\longrightarrow} \underset{\operatorname{tr}}{\lim } B\left(\frac{N H \cap F_{i}}{H}\right)_{+p} .
$$

In order to show $\operatorname{tr}$ is an equivalence, we need to understand how far off $\bigcup_{i}\left(N H \cap F_{i}\right)$ is from $N H$.

Lemma 2.7. $N H=\left(\bigcup_{i}\left(N H \cap F_{i}\right)\right) \cdot(N H \cap T) \quad\left(H \subset F_{i_{0}}\right)$.

Proof. Let $z \in N H$. We can find $\tilde{z} \in \widetilde{F}$ such that $\tilde{z}^{-1} z \in T$. Since $z H z^{-1}=$ $H$ and $\widetilde{F}, H \subset F_{i_{0}}$, an easy calculation shows that $\left(\tilde{z}^{-1} z\right)^{p^{m}} \in N H \cap T$ for some $m$ depending only on $i_{\circ}$. Therefore, as in Lemma 2.5, we can write $\tilde{z}^{-1} z$ as $y_{1} y_{2}$, where $y_{1}, y_{2} \in T, y_{1}$ has order a power of $p$, and $y_{2} \in N H \cap T$. So $z=\tilde{z}\left(\tilde{z}^{-1} z\right)=\tilde{z} y_{1} y_{2}, z, y_{2} \in N H \Rightarrow \tilde{z} y_{1} \in N H$. But notice that $\tilde{z} y_{1} \in F_{k}$ for some large $k$. We are done.

We now have the following map of extensions:

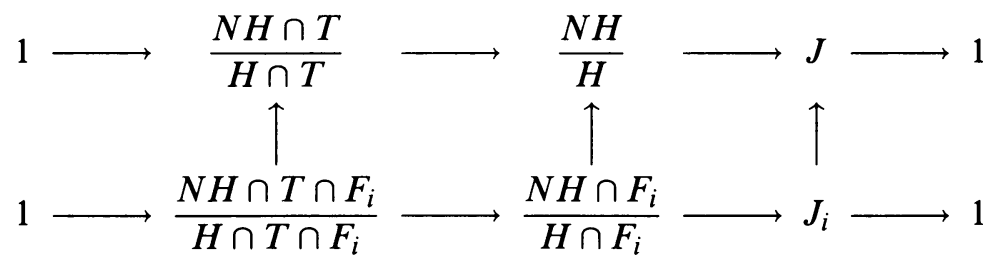

Of course $J$ is finite since $N H / N H \cap T$ is finite.

Corollary 2.8. For sufficiently large $i, J_{i} \rightarrow J$ is an isomorphism.

Proof. For injectivity, suppose $x \in N H \cap F_{i}$ such that $x h=z \in N H \cap T$ for some $h \in H$. If $H \subset F_{i}$, then $z \in F_{i}$ and thus $z \in N H \cap T \cap F_{i}$.

For surjectivity, since $J$ is finite, it suffices to show for each $z \in J$ that there exists $z^{\prime} \in J_{i}$ which maps to $z$ for some $i$. Let $\bar{y} \in N H / H$ map to $z$. By Lemma 2.7, we can write $y=y^{\prime} h$, where $y^{\prime} \in N H \cap F_{i}$ and for some $i$ $h \in N H \cap T$. Then $\bar{y}^{\prime} \in N H \cap F_{i} / H$ has the same image as $\bar{y} \in N H / H$ in $J$.

We are ready to prove 


\section{Proposition 2.9.}

$$
B W_{H}^{\mathrm{Ad} W_{H}} \stackrel{\operatorname{tr}}{\longrightarrow} \underset{i}{\lim } B\left(\frac{N H \cap F_{i}}{H}\right)_{+p}
$$

is an equivalence.

Remark. This will prove Proposition 2.1 and hence Theorem 1.1.

Proof. Following Nishida [13], it is sufficient to check that

$$
\underset{\mathrm{tr}^{*}}{\lim } \bar{H}^{*}\left(B\left(\frac{N H \cap F_{i}}{H}\right)_{+}, Z_{p}\right) \rightarrow \bar{H}^{*}\left(B W_{H}^{\mathrm{Ad} W_{H}}, Z_{p}\right)
$$

is an isomorphism.

Suppose we are in the following situation. Let

$$
1 \rightarrow G_{1} \rightarrow G_{2} \rightarrow G_{3} \rightarrow 1
$$

be an extension of groups. Consider the space $E G_{3} \times_{G_{3}}\left(E G_{2}\right) / G_{1}$, where all actions are from the left and $G_{3} \cong G_{2} / G_{1}$ acts on $\left(E G_{2}\right) / G_{1}$ via the action of $G_{2}$ on $E G_{2}$. Since $E G_{2}$ is $G_{2}$ free, $\left(E G_{2}\right) / G_{1}$ is $G_{2} / G_{1}$ free. This implies that $E G_{3} \times{ }_{G_{3}}\left(E G_{2}\right) / G_{1}$ is equivalent to $\left(\left(E G_{2}\right) / G_{1}\right) / G_{3}=\left(E G_{2}\right) / G_{2}$.

Suppose further that we have the following map of extensions:

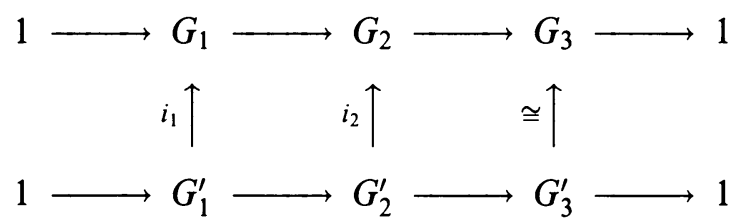

where $i_{1}, i_{2}$ are inclusions. We want to understand the $\operatorname{transfer} B G_{2+} \stackrel{\tau}{\longrightarrow}$ $B G_{2+}^{\prime}$. Recall we have a pretransfer $\tilde{\tau}: S^{0} \rightarrow\left(G_{2} / G_{2}^{\prime}\right)_{+}$, where $\tilde{\tau}$ is a $G_{2}$ map. Taking the smash product with $E G_{2+}$ and the $G_{2}$ orbit yields:

$$
\tau: E G_{2} / G_{2+} \stackrel{1 \wedge_{G_{2}} \bar{\tau}}{\longrightarrow} E G_{2+} \wedge_{G_{2}} G_{2} / G_{2+}^{\prime} \cong E G_{2} / G_{2+}^{\prime} \text {. }
$$

We claim that the following diagram commutes:

$$
\begin{array}{ccc}
E G_{3+} & \wedge_{G_{3}} E G_{2} / G_{1+} \stackrel{1 \wedge_{G_{3} \tau^{\prime}}}{\longrightarrow} E G_{3+} & \wedge_{G_{3}} E G_{2} / G_{1+}^{\prime} \\
\text { proj } \downarrow \cong & \cong \downarrow \text { proj } \\
E G_{2} / G_{2+} & \stackrel{\tau}{\longrightarrow} & E G_{2} / G_{2+}^{\prime}
\end{array}
$$

where $\tau^{\prime}$ is the $G_{3}$ equivariant transfer associated to $G_{1}^{\prime} \hookrightarrow G_{1}$.

Observe that $G_{2}^{\prime} \cap G_{1}=G_{1}^{\prime}$ and hence $G_{1} / G_{1}^{\prime} \cong G_{2} / G_{2}^{\prime}$ as $G_{1}$ spaces. If $\bar{\tau}^{\prime}$ is the pretransfer associated to $\tau^{\prime}$, then this observation implies that $\tilde{\tau}^{\prime}=\tilde{\tau}$. Hence the diagram commutes.

Now, using $1 \wedge_{G_{3}} \tau^{\prime}$, we can filter $E G_{3+}$ by skeleta to get a map of spectral sequences with $E_{2}$ terms:

$$
\bar{H}^{*}\left(B G_{3}, \bar{H}^{*}\left(B G_{1}^{\prime}, Z_{p}\right)\right) \rightarrow \bar{H}^{*}\left(B G_{3}, \bar{H}^{*}\left(B G_{1}, Z_{p}\right)\right)
$$

converging to

$$
\bar{H}^{*}\left(B G_{2}^{\prime}, Z_{p}\right) \stackrel{\tau^{*}}{\longrightarrow} \bar{H}^{*}\left(B G_{2}, Z_{p}\right)
$$


Now, similarly, we have the following maps of spectral sequences when we use dimension shifting pretransfers

$$
S^{0} \rightarrow \Sigma^{-A} F_{+},
$$

where $F=G_{2} / G_{2}^{\prime}$ and $A$ is the adjoint representation of $F$ derived from $\{e\}$.

Now rewrite $(2.8 .1)$ as

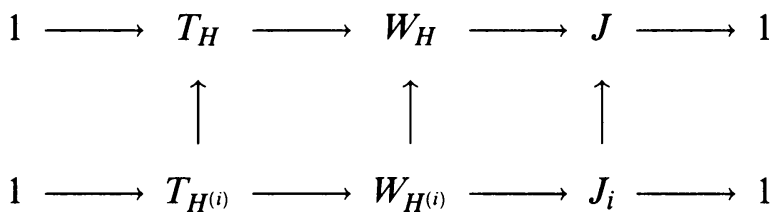

Applying the previous discussion to (2.9.1) yields a map of spectral sequences with $E_{2}$ terms:

$$
\bar{H}^{*}\left(J_{i}, \bar{H}^{*}\left(T_{H^{(i)}}, Z_{p}\right)\right) \stackrel{\tau^{*}}{\longrightarrow} \bar{H}^{*}\left(J, \bar{H}^{*}\left(B\left(T_{H}\right)^{\operatorname{Ad}\left(T_{H}\right)}, Z_{p}\right)\right)
$$

converging to

$$
\bar{H}^{*}\left(W_{H^{(i)}}, Z_{p}\right) \stackrel{\tau^{*}}{\longrightarrow} \bar{H}^{*}\left(B W_{H}{ }^{\text {Ad } W_{H}}, Z_{p}\right),
$$

where $i \geq M$ for some large $M$ so that $J \cong J_{i}$. Since the maps in the spectral sequence are compatible as we vary $i$, it would be sufficient to show that

$$
\underset{\tau^{*}}{\lim } \bar{H}^{*}\left(J_{i}, \bar{H}^{*}\left(T_{H^{(i)}}, Z_{p}\right)\right) \stackrel{\tau^{*}}{\longrightarrow} \bar{H}^{*}\left(J, \bar{H}^{*}\left(B\left(T_{H}\right)^{\operatorname{Ad}\left(T_{H}\right)}, Z_{p}\right)\right)
$$

is an isomorphism. But

$$
\underset{\tau^{*}}{\lim } \bar{H}^{*}\left(J_{i}, \bar{H}^{*}\left(T_{H^{(i)}}, Z_{p}\right)\right)=\bar{H}^{*}\left(J_{i}, \underset{\tau^{*}}{\lim } \bar{H}^{*}\left(T_{H^{(i)}}, Z_{p}\right)\right) .
$$

So we are reduced to proving that

$$
\left.\underset{\tau^{*}}{\lim } \bar{H}^{*}\left(T_{H^{(i)}}, Z_{p}\right) \stackrel{\tau^{*}}{\longrightarrow}\left(B\left(T_{H}\right)^{\operatorname{Ad}\left(T_{H}\right)}, Z_{p}\right)\right)
$$

is an isomorphism. Notice that $T_{H}$ is a compact abelian group. Hence, $T_{H}=$ $C \times T^{k}$ for some finite abelian group $C$ and a torus of rank $k, T^{k}$. But $T_{H^{(i)}}=C^{(p)} \times\left(T_{Q_{p}}^{k} \cap I_{n(i)}\right)$ for sufficiently large $i$, where $C^{(p)}$ is the unique $p$-Sylow subgroup of $C$ and $n(i)$ is an increasing function of $i$. Since we have cohomology in the $Z_{p}$ coefficient, it suffices to verify that

$$
\underset{\tau^{*}}{\lim } \bar{H}^{*}\left(T_{Q_{p}}^{k} \cap I_{n(i)}, Z_{p}\right) \stackrel{\tau^{*}}{\longrightarrow} \bar{H}^{*}\left(B T^{k \operatorname{Ad} T^{k}}, Z_{p}\right)
$$

is an isomorphism. Since it is well known that (cf. [13, Proposition 3.2])

$$
\underset{r}{\lim } \bar{H}^{*}\left(B Z_{p^{r}}, Z_{p}\right) \stackrel{\tau^{*}}{\longrightarrow} \bar{H}^{*}\left(\Sigma B S^{1}, Z_{p}\right)
$$

is àn isomorphism, we are done. 


\section{SOME GENERALIZATIONS}

In this section, we begin by proving an easy splitting result generalizing Theorem 1.1 to other conjugacy classes of $p$-subgroups of $G$. Afterward, we shall consider those conjugacy classes of closed infinite subgroups and verify the injection result of Theorem 3.12.

Our first aim is to prove

Theorem 3.1. Let $G$ be a compact Lie group and $p$ be a fixed prime. Suppose $H$ is a finite p-subgroup of $G$; then $B W_{H} \mathrm{Ad}_{H} \hat{p}$ is a summand of $D B G_{+} \hat{p}$ under $\Phi$. Furthermore, the finite wedge sum of any such spectra splits from $D B G_{+} \widehat{p}$.

We first give some preliminary results. As before, we continue to assume $N$ is an extension of a torus $T$ by a $p$-group unless stated otherwise.

Let $\mathscr{R}_{i}$ denote the wedge of those summands of $\left(S^{0}\right)^{F_{i}} \hat{p}$ whose corresponding subgroup $(H)$ is such that $H \cap T \not \subset F_{i-1} \cap T$. Denote by $\mathscr{S}_{i}$ the wedge of those summands in $\left(S^{0}\right)^{F_{i}} \hat{p}$ that does not belong to $\mathscr{R}_{i}$.

Lemma 3.2. There is a cofiber sequence

$$
{\underset{\lim }{i}}_{i} \mathscr{R}_{i} \rightarrow \underset{i}{\lim }\left(S^{0}\right)^{F_{i}} \stackrel{p}{\rightarrow} \underset{i}{\lim } \mathscr{S}_{i} .
$$

Proof. Recall we have $I_{n}=\left\{x \in T \mid x^{p^{n}}=1\right\}$. Since $I_{n}$ is normal in $N$, summands of $\mathscr{R}_{i}$ will map to summands of $\mathscr{R}_{i-1}$ under the map $\left(S^{0}\right)^{F_{i}} \rightarrow$ $\left(S^{0}\right)^{F_{i-1}}$. This gives rise to the following diagram:

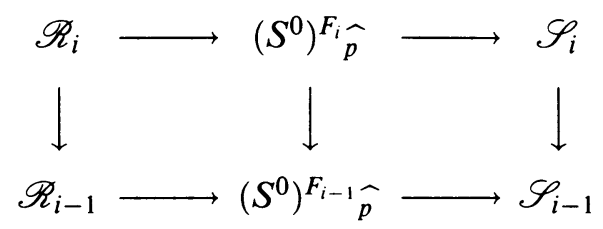

Here the right vertical map will collapse to the base point of $\mathscr{S}_{i-1}$ those summands of $\mathscr{S}_{i}$ whose corresponding subgroup $(H)$ is such that $H \cap T \not \subset F_{i-2} \cap T$. Taking the homotopy inverse limit of the diagram will give us the desired cofiber sequence.

\section{Corollary 3.3.}

$$
\lim _{i} \mathscr{S}_{i}=\prod_{\substack{(H) \subset N \\ H \text { a finite } p \text {-group }}} B W_{H}{ }^{\mathrm{Ad} W_{H}} \hat{p} .
$$

Remark. $\Pi$ denotes the product of spectra (cf. [1, Part III]).

Proof. We have the family $\mathscr{I}_{n}=\left\{H \mid H \cap T \subset I_{n}\right\}$. Notice that given $(H) \in$ $\mathscr{I}_{n}-\mathscr{I}_{n-1}$, then $\left(H^{\alpha} \cap F_{m}\right) \in \mathscr{I}_{n}-\mathscr{I}_{n-1}$ for all $m$ sufficiently large. It follows from Lemma 3.2 that

$$
\varliminf_{i}^{\lim _{i}} \mathscr{S}_{i}=\prod_{n=0}^{\infty}{\underset{i m}{i}}_{i}\left(E\left(\mathscr{S}_{n}, \mathscr{I}_{n-1}\right)_{+}\right)^{F_{i}} \hat{p} .
$$

This, combined with Proposition 2.1, gives the desired result. 
Corollary 3.4. Let $\mathscr{C}$ be a finite collection of conjugacy classes of p-subgroups in $N$. Then the following map splits:

$$
\Phi: \bigvee_{(H) \in \mathscr{C}} B W_{H}{\text { Ad } W_{H}}_{p} \rightarrow \underset{i}{\lim }\left(S^{0}\right)^{F_{i}} \hat{p} \cong D B N_{+} \widehat{p} .
$$

Proof. Let $\mathscr{F}$ be the family of all conjugacy classes of finite $p$-subgroups of $N$. Consider the map

$$
\left(E \mathscr{F}_{+}\right)^{N} \hat{p} \stackrel{\gamma}{\longrightarrow} \underbrace{\lim }_{i}\left(S^{0}\right)^{F_{i}} \underset{p}{\rightarrow} \underset{i}{\lim } \mathscr{S}_{i}
$$

Corollary 3.3 says this is the inclusion of wedges of spectra into products of spectra. Since $\mathscr{C}$ is finite, we therefore have the desired result.

Proof of Theorem 3.1. Let $W_{p}$ be a $p$-Sylow subgroup of the Weyl group $W=$ $N / T$ and let $N_{p}$ be its preimage in $N$. Suppose $H$ is any closed subgroup of $G$ with $\pi_{0}(H)$ a $p$-group. From Bredon [3], we have $\chi\left(\left(N_{p} \backslash G\right)^{H}\right) \equiv \chi\left(N_{p} \backslash G\right)$ $\bmod p$. Since $\chi\left(N_{p} \backslash G\right) \not \equiv 0 \bmod p$, this implies that $\left(N_{p} \backslash G\right)^{H}$ is not empty. In particular, $H$ is conjugate to a subgroup of $N_{p}$. Furthermore, he showed that the $N H$ orbit of $\left(N_{p} \backslash G\right)^{H}$ is finite. Let $\left\{g_{i}\right\}$ be a set of representatives. We then have

$$
\left(N_{p} \backslash G\right)^{H}=\coprod_{i} N H \cap N_{p}^{g_{i}^{-1}} \backslash N H .
$$

Since $\chi\left(\left(N_{p} \backslash G\right)\right) \not \equiv 0 \bmod p$, this says $\chi\left(N H \cap N_{p}^{g_{i}^{-1}} \backslash N H\right) \not \equiv 0 \bmod p$ for some $g_{i}$. In other words, $\chi\left(N H^{g_{i}} \cap N_{p} \backslash N H^{g_{i}}\right) \not \equiv 0 \bmod p$.

Consider the following commutative diagram:

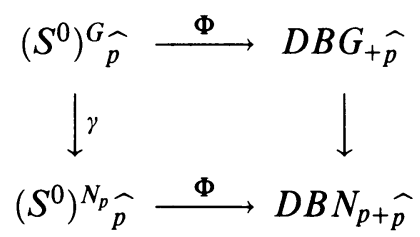

Let $K$ be a $p$-subgroup of $N_{p}$. Recall that, under $\gamma$, the summand corresponding to $(K)$ maps to a finite number of summands corresponding to $\left\{\left(K^{g_{\alpha}^{(i)}} \cap N_{p}\right)\right\}$ which are also $p$-subgroups of $N_{p}$. By Corollary 3.4, it suffices to check $\gamma$ is a splitting map.

Now $K_{\alpha}^{g_{\alpha}^{(i)}} \cap N_{p}$ is not a conjugate of $K$ in $N_{p}$ unless $\bar{g}_{\alpha}^{(i)} \sim \overline{1} \in L=$ $N_{p} \backslash G / N K$. By Propositions 1.12 and 2.6, the map between the summands $(K)$ of $\left(S^{0}\right)^{G} \hat{p}$ and $(K)$ of $\left(S^{0}\right)^{N_{p}} \widehat{p}$ is the bundle transfer:

$$
B W_{K}^{\mathrm{Ad} W_{K}} \stackrel{\mathrm{tr}}{\longrightarrow} B W_{K^{N}} \mathrm{Ad} W_{K^{N}} \hat{p},
$$

where $W_{K^{N}}=\left(N K \cap N_{p}\right) / K$. Therefore we see that $B W_{K} \mathrm{Ad}^{W_{K}} \hat{p}$ is a summand of $D B G_{+} \hat{p}$ if $\operatorname{tr}$ has a retraction.

From Lemma 1.2 and the previous calculation of the Euler characteristics, this holds in particular for $K=H^{g_{i}}$.

Now let $\mathscr{C}$ be any finite collection of conjugacy classes of $p$-subgroups in $G$. Without loss of generality, we may assume each representation $H$ of its conjugacy class is chosen so that

$$
H \subset N_{p} \quad \text { and } \quad \chi\left(N H \cap N_{p} \backslash N H\right) \not \equiv 0 \bmod p .
$$


Let $\mathscr{C}^{\prime}$ be a collection of such representatives.

We want to show that

$$
\bigvee_{H \in \mathscr{C}^{\prime}} B W_{H} \mathrm{Ad}^{\mathrm{Ad} W_{H}} \hat{p} \stackrel{\gamma}{\longrightarrow} \bigvee_{\substack{(K) \subset N_{p} \\ K \text { a } p \text {-group }}} B W_{K^{N}}{ }^{\mathrm{Ad} W_{K^{N}}} \hat{p}
$$

is a splitting map. This would prove the second part of the proposition. For convenience, we may suppose that if $(K)=(H)$ in $N_{p}$ for $H \in \mathscr{C}^{\prime}$, then in fact $K=H$.

For each $H \in \mathscr{C}^{\prime}$, consider the retraction of tr given specifically in Lemma 1.2 , denoted by $\operatorname{ret}_{H}$. Let $\Theta$ be the map

$$
\bigvee_{\substack{(K) \subset N_{p} \\ \text { a } p \text {-group }}} B W_{K^{N}} \operatorname{Ad}^{W_{K^{N}}} \hat{p} \rightarrow \bigvee_{(H) \in \mathscr{C}^{\prime}} B W_{H} \mathrm{Ad}^{W_{H}} \hat{p}
$$

given by $\operatorname{ret}_{K}$ if $K \in \mathscr{C}^{\prime}$ and trivial otherwise. We claim that

$$
\bigvee_{H \in \mathscr{C}^{\prime}} B W_{H}^{\mathrm{Ad} W_{H}} \hat{p} \stackrel{\gamma}{\longrightarrow} \bigvee_{\substack{(K) \subset N_{p} \\ K \text { a } p \text {-group }}} B W_{K^{N}}^{\mathrm{Ad} W_{K^{N}}} \hat{p} \stackrel{\Theta}{\longrightarrow} \bigvee_{H \in \mathscr{C}^{\prime}} B W_{H}^{\mathrm{Ad} W_{H}} \hat{p}
$$

is an equivalence. This would then complete the proof.

Define $\mathscr{C}_{i}^{\prime}=\left\{H \in \mathscr{C}^{\prime}|| H \mid \leq i\right\}$. Observe that since $\left|H^{g_{\alpha}^{(i)}} \cap N_{p}\right| \leq|H|$ for all $g_{\alpha}^{(i)} \in L$, we can filter (3.1.1) so that it suffices to prove the equivalence

$$
\begin{aligned}
& \bigvee_{H \in \mathscr{C}_{i}^{\prime}-\mathscr{C}_{i-1}^{\prime}} B W_{H}^{\mathrm{Ad} W_{H}} \stackrel{\gamma}{\rightarrow} \bigvee_{\substack{(K) \subset N_{p} \\
K \text { a p-group } \\
|K|=i}} B W_{K^{N}} \text { Ad } W_{K^{N}} \hat{p} \\
& \stackrel{\Theta}{\rightarrow} \bigvee_{H \in \mathscr{C}_{i}^{\prime}-\mathscr{C}_{i-1}^{\prime}} B W_{H}{ }^{\mathrm{Ad} W_{H}} \hat{p} .
\end{aligned}
$$

Since $\left|H^{g_{\alpha}^{(i)}} \cap N_{p}\right|=|H|$ implies $H^{g_{\alpha}^{(i)}} \cap N_{p}$ is a conjugate of $H$ in $G$, the map $\Theta$ will map trivially on $\left(H^{g_{\alpha}^{(i)}} \cap N_{p}\right)$ unless $\bar{g}_{\alpha}^{(i)} \sim \overline{1} \in L$. Therefore (3.1.2) is a wedge sum of equivalences.

Corollary 3.5. The following map is an injection:

$$
\pi_{*}(\Phi): \bigoplus_{\substack{(H) \\ H \subset G \text { a } p \text {-group }}} \pi_{*}\left(B W_{H}{\text { Ad } W_{H}}_{p}\right) \rightarrow \pi_{*}\left(D B G_{+p}\right) .
$$

We want to extend Corollary 3.5 to conjugacy classes of closed infinite subgroups of $G$. Let $\mathscr{B}_{2}$ be the family of all conjugacy classes of closed subgroups of $N$ and $\mathscr{B}_{1}$ be the family of those conjugacy classes in $N$ which are finite. Observe $E \mathscr{B}_{2+}$ is $N$-equivariantly homotopic equivalent to $S^{0}$.

Lemma 3.6. There is a map of cofiber sequences:

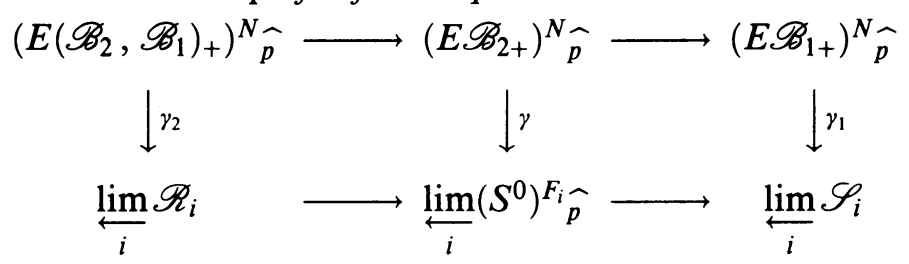

where $\gamma_{1}$ and $\gamma_{2}$ are induced from $\gamma$. 
Proof. We note that given any closed infinite subgroup $H$ of $N, H^{\alpha} \cap T=$ $(H \cap T)^{\alpha}$ has an infinite number of elements. By Corollary 3.3 and Proposition 1.12 , this implies that the summand in $\left(E \mathscr{B}_{2+}\right)^{N} \widehat{p}_{\text {corresponding to }}(H)$ will project trivially into $\lim _{i} \mathscr{S}_{i}$ under $\gamma$.

Of course, it is obvious that $\gamma_{1}$ does not induce an injection on $\pi_{*}$ due to the fact that there are non- $p$-groups in $\mathscr{B}_{1}$. To correct this, let $\mathscr{P}$ be the set $\{(H) \subset N \mid H \quad p$-toral $\}$, and let $\mathscr{P}_{1}=\mathscr{P} \cap \mathscr{B}_{1}$ and $\mathscr{P}_{2}=\mathscr{P} \cap\left(\mathscr{B}_{2}-\mathscr{B}_{1}\right)$. We then have the following map of cofiber sequences:

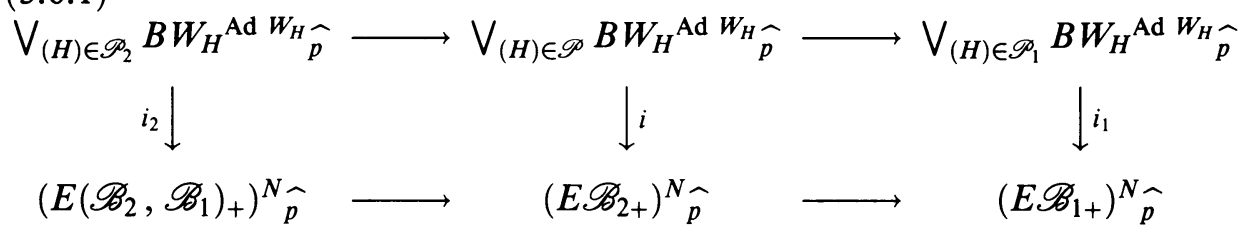

where the $i$ 's are obvious inclusions. Notice that the map $\gamma_{1} \circ i_{1}$ is an inclusion of wedges of spectra into products of spectra.

Suppose we are given a system of $p$-completed spectra $\left\{\mathscr{Z}_{i \hat{p}}\right\}$. Recall from [2] that we have the following exact sequence of groups:

$$
1 \rightarrow \lim ^{1} \pi_{i+1}\left(\mathscr{Z}_{i \hat{p}}\right) \rightarrow \pi_{i}\left(\lim _{\longleftarrow} \mathscr{Z}_{i \hat{p}}\right) \rightarrow \lim \pi_{i}\left(\mathscr{Z}_{i \hat{p}}\right) \rightarrow 1 .
$$

If each $\mathscr{Z}_{i}$ is of finite type, then $\lim ^{1} \pi_{i+1}\left(\mathscr{Z}_{i p}\right)$ would be trivial. Applying this to our situation, it follows that we have the diagram:

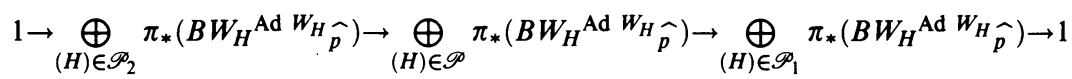

$$
\begin{aligned}
& \gamma_{2} \circ i_{2} \downarrow \quad\left\lfloor\gamma \circ i \quad \left\lfloor\gamma_{i} \circ i_{1}\right.\right. \\
& 1 \rightarrow \quad \underset{i}{\lim } \pi_{*}\left(\mathscr{R}_{i}\right) \quad \rightarrow \quad \underset{i}{\lim } \pi_{*}\left(\left(S^{0}\right)^{F_{i}} \hat{p}\right) \quad \rightarrow \quad \underset{i}{\lim } \pi_{*}\left(\mathscr{S}_{i}\right) \quad \rightarrow 1
\end{aligned}
$$

since $\lim ^{1} \pi_{*}\left(\mathscr{R}_{i}\right)$ is trivial. Therefore in order to show $\pi_{*}(\gamma \circ i)$ is injective, it suffices to show $\pi_{*}\left(\gamma_{2} \circ i_{2}\right)$ is injective by Corollary 3.5 .

To accomplish that, we need to break up $\longleftarrow_{i} \mathscr{R}_{i}$ into pieces more amenable to calculations. This motivates the following

Definition. Recall we have a nested sequence $\left\{F_{i}\right\}$ of finite subgroups of $N$ constructed in Corollary 1.5. Let $H$ be a subgroup of $F_{i}$. We define the type of $H$ to be $|H / H \cap T|$ and the form of $H$ to be $\left|H \cap T / H \cap F_{i-1} \cap T\right|$ which are denoted $t_{i}(H)$ and $f_{i}(H)$, respectively. Note that the definition of type and form is well defined on each conjugacy class in $F_{i}$. For a general $H \subset N$, we can also define its type and form by $t(H)=|H / H \cap T|$ and $f(H)=$ $\stackrel{\lim }{\longrightarrow}_{i}\left|H \cap F_{i} \cap T / H \cap F_{i-1} \cap T\right|$.

Remark. We observe that given $H \subset N, t_{i-1}\left(H \cap F_{i-1}\right) \leq t_{i}\left(H \cap F_{i}\right)$. Similarly, $f_{i}\left(H \cap F_{i}\right) \leq f_{i-1}\left(H \cap F_{i-1}\right)$ via the power map $x \rightarrow x^{p}$.

Lemma 3.7. There exists a finite number of spectra $\left\{\mathscr{U}_{j}\right\}$ and a sequence of maps $\left\{\lambda_{j}\right\}$,

$$
* \stackrel{\lambda_{k}}{\longrightarrow} \mathscr{U}_{k} \stackrel{\lambda_{k-1}}{\longrightarrow} \mathscr{U}_{k-1} \stackrel{\lambda_{k-2}}{\longrightarrow} \cdots \stackrel{\lambda_{0}}{\longrightarrow} \mathscr{U}_{0}=\underset{i}{\lim } \mathscr{R}_{i},
$$


such that the cofiber of each $\lambda_{j}$ is a homotopy inverse limit of a system consisting of all the summands having the same type and form.

Proof. It is clear that there are only a finite number of distinct pairs (type of $H$, form of $H$ ). By the above remark and the fact that $H \subset F_{i}$ will go to $\left\{\left(H^{\alpha} \cap F_{i-1}\right)\right\}$ between consecutive maps in the inverse system, we can filter the system $\left\{\mathscr{R}_{i}\right\}$ so that each successive quotient will be a system having all summands of the same type and form.

Corollary 3.8. Recall $\mathscr{P}$ is the set $\left\{(H) \subset N \mid \pi_{0}(H)\right.$ is a p-group $\}$. Let $\mathscr{P}_{v, \nu}^{\infty}=$ $\{(H) \in \mathscr{P} \mid t(H)=v, f(H)=\nu\}$. Then we can filter $\bigvee_{(H) \in \mathscr{P}_{v, \nu}^{\infty}} B W_{H} \mathrm{Ad}_{W_{H}} \widehat{p}$ in a compatible way with the $\mathscr{U}_{k}$ 's of Lemma 3.7 so that the map between corresponding cofibers is given by

$$
\bigvee_{(H) \in \mathscr{P}_{v, \nu}^{\infty}} B W_{H}^{\text {Ad } W_{H}} \hat{p} \stackrel{\gamma_{v, \nu}}{\longrightarrow} \underbrace{\lim }_{i} \bigvee_{\begin{array}{c}
(H), H \subseteq F_{i} \\
t_{i}(H)=v \\
f_{i}(H)=\nu
\end{array}} B W_{H}^{(i)}+\hat{p}=\underbrace{\lim }_{i} \mathscr{D}_{v, \nu}^{(i)} .
$$

We are reduced to studying the map $\gamma_{v, \nu}$. Let $\Delta$ be the inverse system $\left\{\mathscr{D}_{v, \nu}^{(i)}\right\}$. We first want to study the structure of $\Delta$. Let $\mathscr{G}_{i}$ be a set of representatives of conjugacy classes of subgroups of $F_{i}$ with type $v$ and form $\nu$ such that given any $H^{\prime} \subset F_{i}$ with same type and form, there exist $(H) \in \mathscr{G}_{i}$ and $\alpha \in W$ so that $H^{\prime} \cap T=H^{\alpha} \cap T$. Similarly, we define $\mathscr{G}$ as a set of representatives of conjugacy classes of closed subgroups in $N$ satisfying the same condition. Since $T$ is normal in $N, \mathscr{G}_{i}$ and $\mathscr{G}$ are well defined.

Rewrite $\mathscr{D}_{v, \nu}^{(i)}$ as $\bigvee_{(H) \in \mathscr{Y}_{i}} \mathscr{D}_{H}^{(i)}$, where $\mathscr{D}_{H}^{(i)}$ denotes the wedge of all summands in $\mathscr{D}_{v, \nu}^{(i)}$ whose corresponding representative in $\mathscr{G}_{i}$ is $(H)$. It follows from Proposition 1.12 that $\mathscr{D}_{H}^{(i)}$ will either map to $\mathscr{D}_{H^{\alpha} \cap F_{i-1}}^{(i-1)}$ in $\Delta$ for some $\alpha$ or a map trivially otherwise. Geometrically then, the structure of $\Delta$ is a directed tree $\mathscr{T}$ with each node being represented by some $\mathscr{D}_{H}^{(i)}$.

Lemma 3.9. Let $(H)$ be a conjugacy class in $\mathscr{P}_{v, \nu}^{\infty}$. Then under $\gamma_{v, \nu}$, the summand corresponding to $(H)$ will map to a nontrivial directed path in $\mathscr{T}$. Furthermore, distinct conjugacy classes $(H)$ and $\left(H^{\prime}\right)$ with the same type and form will map into the same directed path in $\mathscr{T}$ under $\gamma_{v, \nu}$ if and only if $H \cap T$ and $H^{\prime} \cap T$ are conjugates in $N$.

Proof. Since $T$ is normal in $N$ and the summand corresponding to $(H)$ will map to summands corresponding to $\left\{\left(H^{\alpha} \cap F_{i}\right)\right\}$ under $\gamma_{v, \nu}$, we only need to show that given a conjugacy class $(H)$ in $\mathscr{P}_{v, \nu}^{\infty}$, we can pick a representative $H_{\circ}$ so that

$$
t\left(H_{\circ}\right)=t_{i}\left(H_{\circ} \cap F_{i}\right) \text { and } f\left(H_{\circ}\right)=f_{i}\left(H_{\circ} \cap F_{i}\right)
$$

for all large $i$. Clearly, by definition, any representative will suffice for the form of $H$. As for the type of $H$, following a proof similar to the proof of Proposition 1.4, we can find a subgroup $H_{Q_{p}}$ of $H$ such that $H_{Q_{p}} \cap T$ consists of all elements in $H \cap T$ of order a power of $p$ and $H_{Q_{p}} / H_{Q_{p}} \cap T \cong H / H \cap T$. By Corollary 1.9, $H_{Q_{p}}$ is conjugate to a subgroup of $N_{Q_{p}}$. Let $\beta \in W$ be such that $H_{Q_{p}}^{\beta} \subset N_{Q_{p}}$. Since $H$ is the closure of $H_{Q_{p}}$ in $N$, this implies that $t\left(H^{\beta}\right)=t_{i}\left(H^{\beta} \cap F_{i}\right)$ for all large $i$. 
It remains to prove that given $(H),\left(H^{\prime}\right) \in \mathscr{P}_{v, \nu}^{\infty}$ such that $H \cap F_{j} \cap T$ and $H^{\prime} \cap F_{j} \cap T$ are conjugates for all large $j$, then $H \cap T$ and $H^{\prime} \cap T$ are in fact conjugates. We may assume $H$ and $H^{\prime}$ satisfy (3.9.1). Since $N / T$ is finite, there exists $\sigma \in W=N / T$ such that $H \cap F_{j} \cap T=H^{\prime \sigma} \cap F_{j} \cap T$ for infinitely many $j$ 's. Consider the unions

$$
\bigcup H \cap F_{j} \cap T, \quad \bigcup H^{\prime \sigma} \cap F_{j} \cap T .
$$

Since $\pi_{0}(H)$ and $\pi_{0}\left(H^{\prime}\right)$ are $p$-groups, the closure of these unions will give $H \cap T$ and $H^{\sigma \sigma} \cap T$, respectively. Hence we have the equality $H \cap T=H^{\prime \sigma} \cap$ $T$.

Remark. From now on, we will assume the representative of each conjugacy class in $\mathscr{P}_{v, \nu}^{\infty}$ is chosen to satisfy (3.9.1).

Lemma 3.10. For each $(H) \in \mathscr{P}_{v, \nu}^{\infty}$, let $J_{H}$ be the induced directed path in $\mathscr{T}$ given by the representative $\left(H \cap F_{i}\right) \in \mathscr{G}_{i}$ at each level $i$. Then the canonical map

$$
\bigvee_{\substack{(H) \in \mathscr{G} \\ J_{H}}} \underset{J_{H}}{\lim } \mathscr{D}_{H \cap F_{i}}^{(i)} \rightarrow \underset{i}{\lim } \mathscr{D}_{v, \nu}^{(i)}
$$

induces an injection on $\pi_{*}$.

Proof. Given $(H) \in \mathscr{G}$, we claim that there exists an integer $r$ such that $H^{\prime} \cap$ $F_{i} \cap T$ is not a conjugate of $H \cap F_{i} \cap T$ for all $i \geq r$ if $\left(H^{\prime}\right) \neq(H) \in \mathscr{G}$. If not, then it is immediate that we may assume $H \cap F_{i} \cap T=H^{\prime} \cap F_{i} \cap T$. Let $(H \cap T)$ 。 and $\left(H^{\prime} \cap T\right)$ 。 be the components of 0 in $H \cap T$ and $H^{\prime} \cap T$, respectively. Since form $=\nu$, we can write $H \cap T=C_{\circ} \times T^{\nu}$ and $H^{\prime} \cap T=C_{\circ}^{\prime} \times\left(T^{\prime}\right)^{\nu}$, where the $C$ 's are cyclic groups.

Suppose $C_{\circ} \subset I_{n}$. We may choose $n$ so that $\left|C_{\circ}\right| \leq p^{n}$. Pick $r$ such that $F_{r} \cap T \supset I_{n+1}$. This $r$ will do the job. We have two cases: $T^{\nu}=T^{\prime \nu}$ or $T^{\nu} \neq T^{\prime \nu}$. In the first case, the equation $\left|H \cap F_{i} \cap T\right|=\left|H^{\prime} \cap F_{i} \cap T\right|$ implies that $C_{\circ}^{\prime} \cap F_{i}$ contains only elements of order less than $p^{n}$. Hence, $C_{\circ}^{\prime} \subset I_{n}$ and $H \cap T=H^{\prime} \cap T$. In the second case, since $T^{\nu} \neq T^{\prime \nu},\left|C_{\circ}^{\prime} \cap F_{i}\right| \geq p^{m}$, where $I_{m}=F_{i} \cap T$. Hence,

$$
\left|H^{\prime} \cap F_{i} \cap T\right| \geq\left(p^{m \nu}\right)\left(p^{m}\right) \geq\left(p^{m \nu}\right)\left(p^{n+1}\right) \geqslant\left(p^{m \nu}\right)\left|C_{\circ}\right|=\left|H \cap F_{i} \cap T\right| .
$$

This implies the directed path associated to each $\left(H^{\prime}\right) \neq(H) \in \mathscr{G}$ will not intersect $J_{H}$ from the level $r$ onward. Since $\mathscr{G}$ is countable, we can order $\mathscr{G}$ as $\left\{\left(H_{1}\right),\left(H_{2}\right), \ldots\right\}$. Choose integer $r_{i}$ for each $\left(H_{i}\right)$ as above. Without loss of generality, we may assume $r_{i} \geq r_{j}$ if $i \geq j$. For each $i$, define $\mathscr{H}_{i}=\left\{(H) \subset F_{i} \mid H \cap F_{r_{j}} \sim H_{j} \cap F_{r_{j}} \in \mathscr{G}_{r_{j}}\right.$, for some $j$ such that $\left.i \geq r_{j}\right\}$. Let

$$
\mathscr{D}_{v, \nu}^{(i)^{\prime}}= \begin{cases}\bigvee_{(H) \in \mathscr{K}_{i}} B W_{H+\hat{p}} & \text { if } \mathscr{K}_{i} \text { is nonempty, } \\ * & \text { otherwise. }\end{cases}
$$

Notice that $\mathscr{D}_{v, \nu}^{(i)^{\prime}}$ consists of those summands in $\mathscr{T}$ which go to $\mathscr{D}_{H_{j} \cap F_{r_{j}}}^{\left(r_{j}\right)}$ for some $j$ such that $i \geq r_{j}$. Form the inverse system $\left\{\mathscr{D}_{v, \nu}^{(i)^{\prime}}\right\}$ where the maps are induced from those of $\Delta$. This gives a canonical map of systems $\left\{\mathscr{D}_{v, \nu}^{(i)}\right\} \rightarrow$ $\left\{\mathscr{D}_{v, \nu}^{(i)^{\prime}}\right\}$. It is clear that $\lim _{i} \mathscr{D}_{v, \nu}^{(i)^{\prime}}$ is a product of homotopy inverse limits, 
and the composite

$$
\bigvee_{(H) \in \mathscr{G}} \underset{J_{H}}{\stackrel{\lim }{\leftrightarrows}} \mathscr{D}_{H \cap F_{i}}^{(i)} \rightarrow \underset{i}{\lim } \mathscr{D}_{v, \nu}^{(i)} \rightarrow \underset{i}{\lim } \mathscr{D}_{v, \nu}^{(i)^{\prime}}
$$

maps distinct wedge summands of the source into distinct product factors of the target. However, upon taking $\pi_{*}$, each wedge summand maps injectively into its target since an inverse limit of inclusions of groups is an inclusion. Therefore this gives the desired result.

Proposition 3.11. Fix a $(H) \in \mathscr{P}_{v, \nu}^{\infty}$. The following map induces an injection on $\pi_{*}$ :

$$
\gamma_{v, \nu}^{(H)}: \bigvee_{\substack{\left(H^{\prime}\right) \\\left(H^{\prime}\right) \sim(H) \in \mathscr{G}}} B W_{H^{\prime}}{ }^{\text {Ad } W_{H^{\prime}} \hat{p}} \rightarrow \underset{J_{H}}{\lim } \mathscr{D}_{H \cap F_{i}}^{(i)} .
$$

Here $\gamma_{v, \nu}^{(H)}$ is the restriction of $\gamma_{v, \nu}$.

Proof. We first prove the proposition for the summand corresponding to $(H)$. For each $i$, define

$$
\begin{aligned}
\mathscr{Z}_{i}=\left\{(K) \subset F_{i} \mid(K)\right. & \sim\left(H \cap F_{i}\right) \in \mathscr{G}_{i} \text { and }(K) \\
& \text { is a conjugate of } \left.\left(H^{t} \cap F_{i}\right) \text { for some } t \in T\right\} .
\end{aligned}
$$

Consider the cofiber sequence

$$
\bigvee_{(K) \in \mathscr{X}_{i}} B W_{K}^{(i)}+\hat{p} \rightarrow \mathscr{D}_{H \cap F_{i}}^{(i)} \rightarrow \mathscr{F}_{i}
$$

where $\mathscr{F}_{i}$ is the cofiber of the left arrow. Notice that under $F_{i} \hookrightarrow F_{i+1}$, the $\mathscr{Z}_{i}$ 's are compatible by Proposition 1.11. Since $\gamma_{v, \nu}^{(H)}$ restricted to $B W_{H}{ }^{\mathrm{Ad} W_{H}} \hat{p}$ actually factors through the homotopy inverse limit $\mathfrak{\operatorname { l i m }}_{i} \bigvee_{(K) \in \mathscr{X}_{i}} B W_{K}^{(i)}+\widehat{p}$, it suffices to check that

$$
\gamma_{v, \nu}^{(H)}: B W_{H}{ }^{\text {Ad } W_{H}} \widehat{p} \rightarrow \underset{i}{\lim } \bigvee_{(K) \in \mathscr{Z}_{i}} B W_{K}^{(i)}+\widehat{p}
$$

induces an injection on $\pi_{*}$.

Consider the double coset $F_{i} \backslash^{N} / N H$. Let $L_{i}=\left\{g \in F_{i} \backslash^{N} / N H \mid t_{i}\left(H^{g} \cap F_{i}\right)\right.$ $\left.=v, f_{i}\left(H^{g} \cap F_{i}\right)=\nu\right\}$. We define an equivalence relation on $L_{i}$ by $g_{1} \sim g_{2}$ if $H^{g_{1}} \cap F_{i}$ is a conjugate of $H^{g_{2}} \cap F_{i}$ in $F_{i}$. It is easy to check that this equivalence relation is well defined. By definition, each $(K) \in \mathscr{Z}_{i}$ gives rise to an equivalence class in $L_{i}$.

We claim that for all sufficiently large $i$, the equivalence relation induces a well-defined map $j: L_{i} / \sim \rightarrow L_{i+1} / \sim$ coming from $F_{i} \hookrightarrow F_{i+1}$. That is if $g_{1} \sim g_{2} \in L_{i}$, then $j\left(g_{1}\right) \sim j\left(g_{2}\right) \in L_{i+1}$. We may assume $g_{1}, g_{2} \in T$. Since $g_{1}, g_{2}$ are chosen so that $t(H)=t_{i}\left(H^{g_{1}} \cap F_{i}\right)=t_{i}\left(H^{g_{2}} \cap F_{i}\right)$, we have

$$
H^{g_{k}}=H^{g_{k}} \cap F_{i} \cdot H^{g_{k}} \cap T=H^{g_{k}} \cap F_{i} \cdot H \cap T, \quad k=1,2 .
$$

Since $H \cap F_{Q_{p}} \cap T$ is dense in $H \cap T$ and $N / T$ is finite, we can find some sufficiently large $m_{\circ}$ such that if $\sigma \in N$ normalizes $H \cap F_{m_{\circ}} \cap T$, then $\sigma$ also normalizes $H \cap T$. So pick $i \geq m_{\circ}$. If $\left(H^{g_{1}} \cap F_{i}\right)^{\sigma}=H^{g_{2}} \cap F_{i}$ for some $\sigma \in F_{i}$, then this implies that $\left(H^{g_{1}} \cap F_{i}\right)^{\sigma} \cap T=H^{g_{2}} \cap F_{i} \cap T$. But $g_{1}, g_{2} \in T$ and thus 
$\sigma$ normalizes $H \cap T$. Hence, we have $H^{\sigma g_{1}}=H^{g_{2}}$. Consequently, $H^{g_{1}} \cap F_{i+1}$ is a conjugate of $H^{g_{2}} \cap F_{i+1}$ in $F_{i+1}$.

Now the analogue in Lemma 2.3 shows that the summand corresponding to $\left(H^{g} \cap F_{i+1}\right) \in \mathscr{Z}_{i+1}$ will map to summands corresponding to $\left\{\left(H^{\left(g_{k}\right)} \cap F_{i}\right) \in \mathscr{Z}_{i}\right\}$ for all sufficiently large $i$, where $j^{-1}(g)=\left\{g_{1}, g_{2}, \ldots\right\}$. Similarly, using the fact that the types of these groups are all the same, we have the analogue of Lemma 2.5. In summary, we see that it suffices to verify that

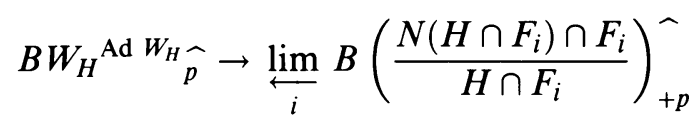

is an equivalence.

A priori, by Proposition 1.12, the maps in the homotopy inverse limit above are compositions of transfers and natural inclusions, i.e.,

$$
\begin{aligned}
& B \frac{N\left(H \cap F_{i+1}\right) \cap F_{i+1}}{H \cap F_{i+1}}+\hat{p} \stackrel{\operatorname{tr}}{\longrightarrow} B \frac{N\left(H \cap F_{i+1}\right) \cap F_{i}}{H \cap F_{i}}+\hat{p} \\
& \stackrel{B i}{\longrightarrow} B \frac{N\left(H \cap F_{i}\right) \cap F_{i}}{H \cap F_{i}}+\hat{p} .
\end{aligned}
$$

However, we want to show that for all sufficiently large $i$,

$$
N H \cap F_{i}=N\left(H \cap F_{i}\right) \cap F_{i} .
$$

If this is indeed the case, then $N\left(H \cap F_{i+1}\right) \cap F_{i}=N H \cap F_{i+1} \cap F_{i}=N\left(H \cap F_{i}\right) \cap F_{i}$ and thus

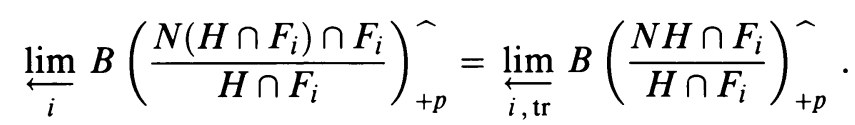

Clearly, $N H \cap F_{i} \subset N\left(H \cap F_{i}\right) \cap F_{i}$ for all $i$. As before, we can find some sufficiently large $m_{\circ}$ so that is $\sigma \in N / T$ normalizes $N \cap F_{i} \cap T$ and $i \geq m_{\circ}$, then $\sigma$ also normalizes $H \cap T$. Since $t(H)=t_{i}\left(H \cap F_{i}\right)$ for all large $i$, the equation $H=H \cap F_{i} \cdot H \cap T$, which holds for such $i$ 's, implies $N\left(H \cap F_{i}\right) \cap F_{i} \subset$ $N H \cap F_{i}$.

Henceforth we want to check the following equivalence:

$$
B W_{H}{\text { Ad } W_{H}}_{p} \stackrel{\operatorname{tr}}{\longrightarrow} \underset{i, \operatorname{tr}}{\lim } B\left(\frac{N H \cap F_{i}}{H \cap F_{i}}\right)_{+p} .
$$

Consider the following map of extensions:

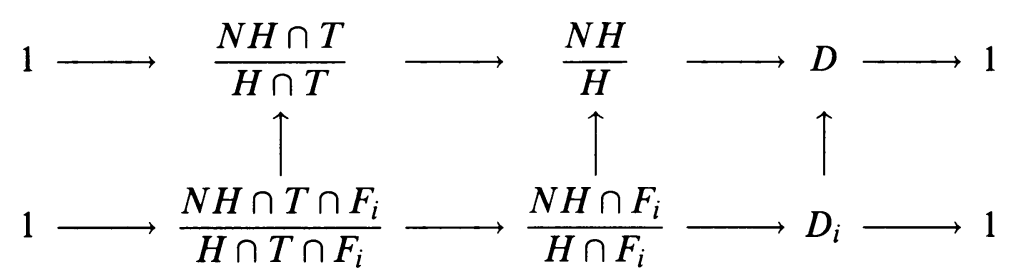

We claim $D_{i} \rightarrow D$ is an isomorphism for sufficiently large $i$. The argument used in Proposition 2.8 would then prove (3.11.1).

It is easy to see $D_{i} \hookrightarrow D$. So suppose $z \in N H$. Write $z=\tilde{z} \cdot t$, where $t \in T$ and $\tilde{z} \in \widetilde{F}$. Pick $i$ so that $t_{i}\left(H \cap F_{i}\right)=t(H)$. This together with $H^{z} \cap F_{i}=H \cap F_{i}$ implies that $t^{p^{k}} \in N H$ for some integer $k$. As in the proof 
of Lemma 2.5, we could write $t=t_{1} \cdot t_{2}$, where $t_{2} \in N H$ and $t_{1}$ is of order a power of $p$. Therefore we have shown surjectivity.

Hence we have proved the injection result for the single summand corresponding to $(H)$. As for other summands, we note that if $\left(H^{\prime}\right) \neq(H)$ and $H^{\prime} \cap T=H \cap T$, then $H^{\prime} \cap F_{i}$ is not a conjugate of $H^{t} \cap F_{i}$ in $F_{i}$ for all sufficiently large $i$ and all $t \in T$. The proof of this fact is just the argument used for showing $j: L_{i} / \sim \rightarrow L_{i+1} / \sim$ is well defined. Now apply the above analysis of $\stackrel{\lim }{\longleftarrow}_{i} \bigvee_{(K) \in \mathscr{Z}_{i}} B W_{K}^{(i)}+\hat{p}$ to ${\underset{\lim }{\longleftarrow}}_{i} \mathscr{F}_{i}$ and combine with Lemma 1.7 to complete the proof.

The generalization of Corollary 3.5 is

Theorem 3.12. The following map is an injection:

$$
\pi_{*}(\Phi): \bigoplus_{\substack{(H) \subset G \\ \pi_{0}(H) \text { a p proup }}} \pi_{*}\left(B W_{H}^{\mathrm{Ad} W_{H}} \hat{p}\right) \rightarrow \pi_{*}\left(D B G_{+} \hat{p}\right) .
$$

Proof. It suffices to show

$$
\bigoplus_{\substack{(H) \subset G \\ \pi_{0}(H) \text { a } p \text {-group }}} \pi_{*}\left(B W_{H} \mathrm{Ad}^{W_{H}} \hat{p}\right) \stackrel{\gamma}{\longrightarrow} \pi_{*}^{N_{p}}\left(S_{p}^{0} \hat{p}\right) \stackrel{\pi_{*}\left(\Phi_{N_{p}}\right)}{\longrightarrow} \pi_{*}\left(D B N_{p+\hat{p}}\right)
$$

is an injection.

By combining Lemmas 3.8 and 3.9 and Proposition 3.11, we notice that the theorem holds for $N_{p}$. Recall that, under $\gamma$, the summand corresponding to $(H)$ of $G$ maps to a finite number of summands corresponding to $\left\{\left(H^{g_{\alpha}^{(i)}} \cap N_{p}\right)\right\}$ of $N_{p}$. As in the proof of Theorem 3.1, we may assume each representative $H$ belongs to $N_{p}$ whenever $(H) \in \mathscr{P}$. Furthermore, a slight modification of the proof given there shows that the map

$$
\bigoplus_{\substack{(H) \subset G \\ \pi_{0}(H) \text { a } p \text {-group }}} \pi_{*}\left(B W_{H}{\text { Ad } W_{H}}_{p}\right) \stackrel{\gamma}{\longrightarrow} \pi_{*}^{N_{p}}\left(S_{p}^{0} \hat{p}\right)
$$

is an injection. Of course, this does not immediately give us what we want.

Let $z$ be a nonzero element in $\pi_{*}^{N_{p}}\left(S_{p}^{0} \hat{p}\right)$. We can rewrite $z$ uniquely as a sum of $x$ and $y$, where $x \neq 0$ comes from summands whose corresponding conjugacy class $(H)$ is such that the dimension of $H$ is maximal possible. Notice the analysis of $\gamma \circ i$ in (3.6.1) could equally well be applied to $\gamma$ itself even though we will not have injections in general. Therefore we observe that the argument presented in the proof of Lemma 3.10 together with Corollary 3.8 actually shows $\pi_{*}\left(\Phi_{N_{p}}\right)(z)=0$ implies $\pi_{*}\left(\Phi_{N_{p}}\right)(x)=0$.

Clearly for $(H) \subset G$ with $\pi_{0}(H)$ a $p$-group, the dimension of $H$ is greater than or equal to the dimension of $H^{g_{\alpha}^{(i)}} \cap N_{p}$ and equality holds only if $\pi_{0}\left(H^{g_{\alpha}^{(i)}} \cap N_{p}\right)$ is a $p$-group. The above discussion then proves $(3.12 .1)$ is an injection.

In our discussion up to now we have not mentioned those conjugacy classes of subgroups of $G$ which are not subconjugates of $N$. The following proposition explains the reason. 
Proposition 3.13. Let $(H)$ be a conjugacy class of $N$. Then under the map

$$
\left(S^{0}\right)^{N} \stackrel{D \tau}{\longrightarrow}\left(S^{0}\right)^{G},
$$

the summand corresponding to $(H)$ of $\left(S^{0}\right)^{N}$ goes to the corresponding $(H)$ of $\left(S^{0}\right)^{G}$ given by a map induced by the inclusion $N_{N} H \hookrightarrow N_{G} H$, where $\tau$ is the $G$-transfer $S^{0} \rightarrow G / N_{+}$.

Proof. From tom Dieck's splitting, for a given $f \in \pi_{*}\left(B W_{H}{ }^{\text {Ad } W_{H}}\right)$, the image of $f$ in $\pi_{*}^{G}\left(S^{0}\right)$ is the following composite:

$$
\begin{aligned}
f_{1}: & S^{0} \stackrel{f}{\longrightarrow} B W_{H}{ }^{\mathrm{Ad} W_{H}} \stackrel{\tau}{\longrightarrow} N_{+} \wedge_{N_{N} H} B W_{H}{ }^{\mathrm{Ad} W_{H}} \\
& \stackrel{1 \wedge \tau}{\longrightarrow} N_{+} \wedge_{N_{N} H} E W_{H+} \stackrel{\mathrm{pr}}{\longrightarrow} S^{0},
\end{aligned}
$$

where $\mathrm{pr}$ is the obvious projection map. If we identify $\left\{G / N_{+}, S^{0}\right\}_{*}^{G}$ with $\left\{S^{0}, S^{0}\right\}_{*}^{N}$, the above composite becomes:

$$
f_{2}: G / N_{+} \wedge S^{0} \stackrel{1 \wedge f_{1}}{\longrightarrow} G / N_{+} \wedge S^{0} \stackrel{\mathrm{pr}}{\longrightarrow} S^{0} .
$$

Now, under the $G$-transfer $S^{0} \stackrel{\tau}{\longrightarrow} G / N_{+}, f_{2}$ sends to $f_{3}=f_{2} \circ \tau$. Consider the following diagram:

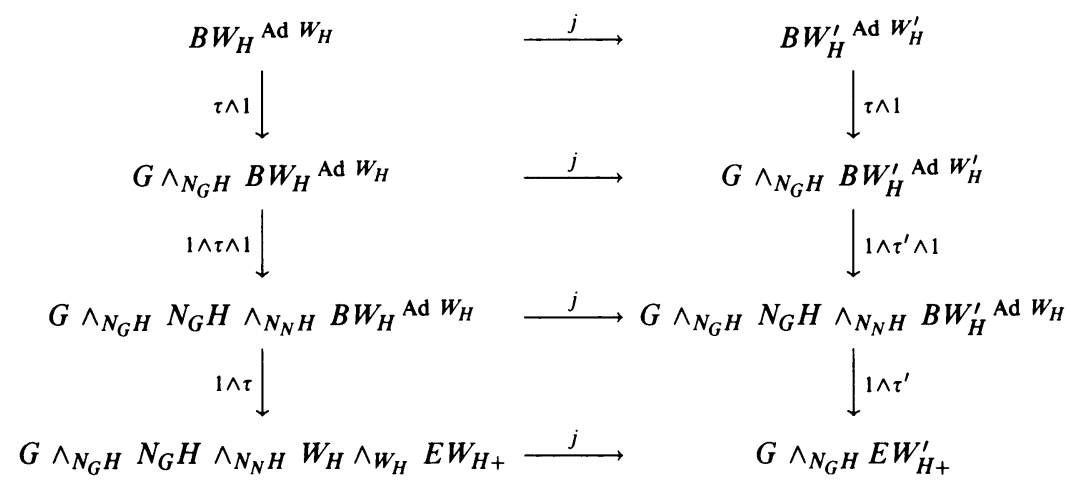

where $W_{H}^{\prime}=N_{G} H / H, \tau\left(\tau^{\prime}\right)$ are appropriate transfers (dimension-shifting transfers), and $j$ is induced from inclusions $\operatorname{Ad} W_{H} \hookrightarrow \operatorname{Ad} W_{H}^{\prime}$ and $W_{H} \hookrightarrow$ $W_{H}^{\prime}$. Since the diagram commutes, we have the desired result.

Notice the same argument applies if we replace $N$ by $N_{p}$ in the proposition. Consider the following commutative diagram:

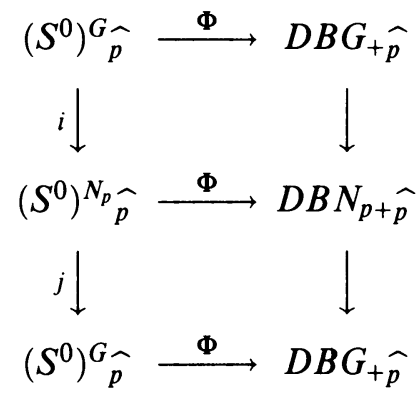

where $i$ is induced from inclusion and $j$ is induced from $\tau: S^{0} \rightarrow G / N_{p+}$. Observe that the vertical composite of the right side is an equivalence. By the 
preceding proposition and Proposition 1.12, the summand corresponding to ( $H$ ) will map to summands of the form $H^{\alpha} \cap N_{p}$ under the composite $j \circ i$. If $(H) \not \subset\left(N_{p}\right)$, then $\left(H^{\alpha} \cap N_{p}\right) \neq(H)$ for any $\alpha$. Therefore we conclude that the results of this section are not far from being optimal.

$$
\text { 4. } G=S O(3)
$$

In this section we want to consider the case when $G=S O(3)$. Of course, the previous sections gave a partial description of $\mathrm{DBSO}(3)_{+}$at all primes. However, we can actually do better and obtain an explicit splitting of $\mathrm{DBSO}(3)_{+}$at the prime 2 .

We note that the proofs of Theorems 1.1 and 3.1 can basically be reduced to the case when $G$ is an extension of a torus by a $p$-group. Therefore we begin by deriving a crude structural theorem of $D B G_{+} \hat{p}$ in that case.

Let $\eta: J_{1} \rightarrow J_{2}$ be a surjection of finite $p$-groups with kernel $K$. We want to understand the induced map $D B J_{2+} \stackrel{D B \eta}{\longrightarrow} D B J_{1+}$. By Segal's conjecture for $p$-groups, this is equivalent to studying $\left(S^{0}\right)^{J_{2}} \rightarrow\left(S^{0}\right)^{J_{1}}$.

As before, we have a commutative diagram:

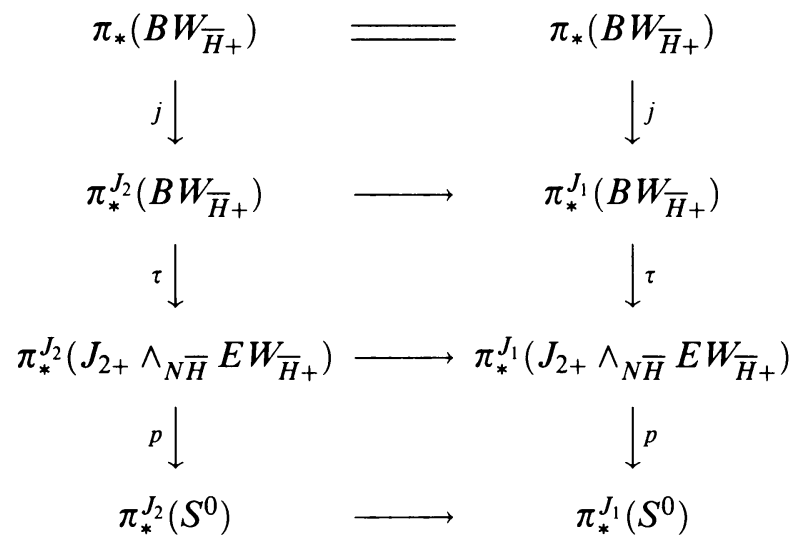

where the horizontal maps are induced from $J_{1} \stackrel{\eta}{\longrightarrow} J_{2}$ and $\bar{H}$ is a subgroup of $J_{2}$. Let $H$ be the preimage of $\bar{H}$ in $J_{1}$.

Lemma 4.1. $J_{2+} \wedge_{N \bar{H}} E W_{\bar{H}+}=J_{1+} \wedge_{N H} E W_{H+}$ as $J_{1}$-spaces.

Proof. Observe that $N(\bar{H})=\overline{N H}$. Hence, $J_{2} / N(\bar{H})=J_{1} / K \times \overline{N H}=J_{1} / N H$ as $J_{1}$-spaces. Since $N(\bar{H}) / \bar{H}=\overline{N H} / \bar{H} \cong N H / H$ and clearly the action of $N H$ on $E W_{\bar{H}}$ is the same as that of $N H$ on $E W_{H}$, we have the lemma.

Corollary 4.2. The summand of $\left(S^{0}\right)^{J_{2}}$ corresponding to $(\bar{H})$ maps under $\eta$ to the summand $(H)$ of $\left(S^{0}\right)^{J_{1}}$ via an equivalence induced from $\eta^{-1}$.

We can apply this simple result to the following special situation. Suppose that

$$
1 \rightarrow T \rightarrow N \rightarrow W \rightarrow 1
$$

is a split extension with $W$ a $p$-group. That is, $N$ is a semidirect product of $W$ and $T$, i.e., $N=W \ltimes T$. Recall $I_{m}=\left\{x \in T \mid x^{p^{m}}=1\right\}$ and let $N_{m}=W \ltimes I_{m}$. We have a surjection $\eta: N_{m+1} \rightarrow N_{m}$ induced from the power 
map $I_{m+1} \stackrel{()^{p}}{\longrightarrow} I_{m}$. Consider the following commutative diagram:

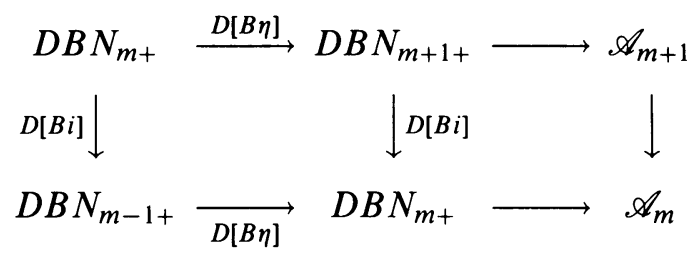

where $i$ is the inclusion $N_{m} \hookrightarrow N_{m+1}$ and $\mathscr{A}_{m}$ is the cofiber of $D[B \eta]$. As a matter of fact, from Corollary 4.2 the map $D[B \eta]$ splits and $\mathscr{A}_{m}$ is a wedge of those summands of $\left(S^{0}\right)^{N_{m}} \hat{p}$ corresponding to $(H)$ such that $I_{1} \not \subset H$. It is clear then under $D[B i], \mathscr{A}_{m+1}$ maps into $\mathscr{A}_{m}$ only. Therefore, the diagram (4.3.1) splits naturally. Taking the homotopy inverse limit with respect to $m$ yields

$$
D B N_{+} \hat{p} \stackrel{D[B \bar{\eta}]}{\longrightarrow} D B N_{+} \widehat{p} \longrightarrow \underset{m}{\lim } \mathscr{A}_{m} .
$$

Here $\bar{\eta}$ is induced from $T \stackrel{()^{p}}{\longrightarrow} T$ and of course the sequence splits. By iterating $D[B \bar{\eta}]$, we get:

$$
\begin{aligned}
& D B N_{+\hat{p}} \stackrel{D\left[B \bar{\eta}^{n+1}\right]}{\longrightarrow} D B N_{+} \longrightarrow \bigvee_{1}^{n+1} \underbrace{\left(\lim _{m} \mathscr{A}_{m}\right)}_{m} \\
& D[B \bar{\eta}] \downarrow \quad \| \\
& D B N_{+\hat{p}} \stackrel{D\left[B \bar{\eta}^{n}\right]}{\longrightarrow} D B N_{+} \longrightarrow \bigvee_{1}^{n} \underset{m}{(\underbrace{\lim }_{m} \mathscr{A}_{m})}
\end{aligned}
$$

where the right vertical map collapses the $(n+1)$ th wedge summand to the base point. Taking the homotopy inverse limit with respect to $n$ yields the split cofiber sequence

$$
{\underset{D[B \bar{\eta}]}{\lim }}_{\left[N_{+}\right.} D B N_{p} \rightarrow D B N_{+\hat{p}} \rightarrow \prod_{1}^{\infty}(\underbrace{\lim }_{m} \mathscr{A}_{m})
$$

Now

$$
\lim _{D[B \bar{\eta}]} D B N_{+} \widehat{p}=D(\underbrace{\lim }_{B \bar{\eta}} \widetilde{B N}_{+})
$$

where $\sim$ denotes the cocompletion at $p$ or equivalently, the smash product with the Moore spectrum $\Sigma^{-1} M\left(Z_{p \infty}\right)$. But

$$
\underset{B \bar{\eta}}{\lim _{\overrightarrow{B N}}} \widetilde{B N}_{+}=\underset{B \bar{\eta}}{\lim }\left(\underset{B i}{\lim } \widetilde{B N}_{m+}\right)=\underset{B i}{\lim }\left(\underset{B \bar{\eta}}{\lim } \widetilde{B N}_{m+}\right)=\underset{B i}{\lim } \widetilde{B W}_{+}=\widetilde{B W}_{+} .
$$

Therefore

$$
D B N_{+} \hat{p}=D B W_{+} \widehat{p} \vee \prod_{1}^{\infty}\left(\underset{m}{\lim } \mathscr{A}_{m}\right)
$$


Proposition 4.3. Let $1 \rightarrow T \rightarrow N \rightarrow W \rightarrow 1$ be an arbitrary extension with $W$ a p-group, $p$ a prime. Then $D B N_{+} \hat{p}$ can be written in the form

$$
D B W_{+} \hat{p} \vee \mathscr{A}^{\prime} \vee \prod_{1}^{\infty} \mathscr{A}
$$

for some p-completed spectra $\mathscr{A}$ and $\mathscr{A}^{\prime}$.

Proof. From Proposition 1.4, there exists a subgroup $\widetilde{F}$ of $N$ such that $\widetilde{F} \cap T$ equals the subgroup $\widetilde{T}$ of $T$ generated by all elements of order $n=|W|$. Corollary 1.5 says we can find a nested sequence $\left\{F_{i}\right\}$ of finite subgroups of $N$ such that $F_{i} / F_{i} \cap T \cong W$ with $\bigcup F_{i} \cap T=T_{Q_{p}}$. Consider the following diagram:

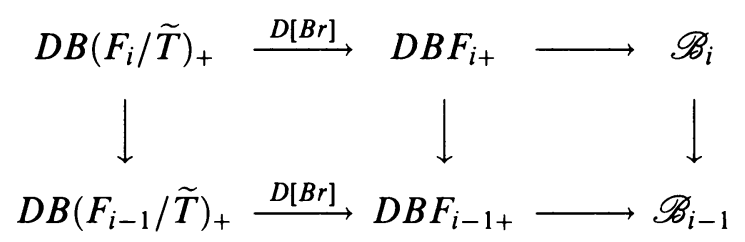

where $r: F_{i} \rightarrow F_{i} / \widetilde{T}$ is the quotient map and the vertical maps are induced from $F_{i-1} \hookrightarrow F_{i}$. By taking the homotopy inverse limit and observing the naturality of the splittings, it is immediate that we have

$$
D B N_{+} \widehat{p}=D B(N / \widetilde{T})_{+} \widehat{p} \vee \underset{i}{\lim } \mathscr{B}_{i} .
$$

However, the extension

$$
1 \rightarrow \frac{T}{\widetilde{T}} \rightarrow \frac{N}{\widetilde{T}} \rightarrow W \rightarrow 1
$$

splits since $\widetilde{F} / \widetilde{T} \cong W$. Therefore, applying the previous discussion to this situation results in the assertion.

We get the following result of Ravenel [14, Theorem 1.11].

\section{Corollary 4.4.}

$$
D B S^{1}+\hat{p}=S_{p}^{0} \widehat{p} \vee \prod_{1}^{\infty} B S^{1 \text { Ad } S^{1}} \hat{p} .
$$

As we shall see later, Proposition 4.3 also recovers a result of Miller and Wilkerson [12].

We are now ready to describe $D B O(2)_{+} \hat{p}$. Recall that the dihedral group $D_{n}$ of order $2 n$ is given by

$$
\operatorname{gp}\left\langle s, t \mid s^{n}=1=t^{2}, t^{-1} s t=s^{-1}\right\rangle, \quad n \geq 1 .
$$

We have the following maps:

$i: D_{2^{n}} \rightarrow D_{2^{n+1}}, \quad i$ the inclusion map given by $s \rightarrow s^{2}, t \rightarrow t$,

$r: D_{2^{n}} \rightarrow D_{2^{n-1}}, \quad r$ the reduction map given by $s \rightarrow s, t \rightarrow t$.

Lemma 4.5. The cofiber $\mathscr{C}_{n}$ of $D[B r]$ is $B D_{2^{n}+\hat{2}} \vee B Z_{2+\hat{2}} \wedge B Z_{2+\hat{2}}$. 
Proof. The conjugacy classes of subgroups of $D_{2^{n}}$ consist of

$$
\begin{aligned}
T_{2^{j}} & =\mathrm{gp}\left\langle s^{2^{n-j}}\right\rangle, & & j=0,1, \ldots, n, \\
D_{2^{j}} & =\mathrm{gp}\left\langle s^{2^{n-j}}, t\right\rangle, & & j=0,1, \ldots, n, \\
D_{2^{j}}^{\prime} & =\mathrm{gp}\left\langle s^{2^{n-j}}, s t\right\rangle, & & j=0,1, \ldots, n-1 .
\end{aligned}
$$

By Corollary $4.2, \mathscr{C}_{n}$, the cofiber of $D[B r]$, is

$$
B W_{T_{1}+\hat{2}} \vee B W_{D_{1}+\hat{2}} \vee B W_{D_{1}^{\prime}+\hat{2}} \text {. }
$$

Now

$$
\begin{aligned}
N T_{2^{j}} & =D_{2^{n}}, \\
N D_{2^{j}} & =D_{2^{j+1}} \quad \text { if } j \neq n, \\
N D_{2^{j}}^{\prime} & =D_{2^{j+1}}^{\prime} \quad \text { if } j \neq n-1, \\
N D_{2^{n-1}}^{\prime} & =D_{2^{n}} .
\end{aligned}
$$

Hence, $\mathscr{C}_{n}=B D_{2^{n}+\hat{2}} \vee B Z_{2+\hat{2}} \vee B Z_{2+\hat{2}}$.

Proposition 4.6.

$$
D B O(2)_{+\hat{2}}=S_{2}^{0} \hat{2} \vee B Z_{2+\hat{2}} \vee \prod_{1}^{\infty} B O(2)^{\mathrm{Ad} O(2)} \hat{2} \vee \prod_{1}^{\infty} B Z_{2+\hat{2}}
$$

Proof. Notice that

$$
{\underset{D[B i]}{\leftrightarrows}}_{\mathscr{C}_{n}}=\underbrace{\lim }_{n}\left(E \mathscr{F}_{+}\right)^{D_{2^{n}}} \hat{2},
$$

where $\mathscr{F}=\{H \subset O(2) \mid H \cap T=1\}$. Therefore, by Proposition 2.1, ${\underset{\lim }{\longleftarrow}}_{D[B i]} \mathscr{C}_{n}$ $=\left(E \mathscr{F}_{+}\right)^{O(2)} \hat{2}$. But then $\mathscr{F}$ only consists of two conjugacy classes, namely (1) and $\left(D_{1}\right)$. Hence, $\stackrel{\lim }{\longleftarrow}_{D[B i]} \mathscr{C}_{n}=B O(2)^{\text {Ad } O(2)} \hat{2} \vee B Z_{2+\hat{2}}$. The rest follows from the discussion after Corollary 4.2.

We record the description of the map $\left(E \mathscr{F}_{+}\right)^{O(2)} \hat{2} \stackrel{\gamma}{\longrightarrow}\left(E \mathscr{F}_{+}\right)^{D_{2}} \hat{2}$ to be needed later. For convenience, we denote by $B D_{1+\hat{2}}$ the summand corresponding to $\left(D_{1}\right)$ in $D B D_{2+\hat{2}}$ and by $B D_{1+\hat{2}}^{\prime}$ the summand corresponding to $\left(D_{1}^{\prime}\right)$.

Lemma 4.7. In $\left(E \mathscr{F}_{+}\right)^{O(2)} \hat{2} \stackrel{\gamma}{\longrightarrow}\left(E \mathscr{F}_{+}\right)^{D_{2}} \hat{2}$, we have

(1) $B O(2)^{\mathrm{Ad} O(2)} \hat{2}$ maps to $B D_{2+\hat{2}}$ by the bundle transfer and is trivial otherwise;

(2) $B Z_{2+\hat{2}}$ maps to $B D_{1+\hat{2}}$ and $B D_{1+\hat{2}}^{\prime}$ by the identity;

(3) $B Z_{2+\hat{2}}$ maps to $B D_{2+\hat{2}}$ by $(-1) \cdot B i$, where $i: Z_{2} \hookrightarrow D_{2}$.

Proof. The proof follows easily from Proposition 1.12.

Given a compact Lie group $G$ with normalizer $N$ of a maximal torus $T$, an element $x \in \pi^{*}(N)$ is stable with respect to $G$ if

$$
\rho^{*}\left(N \cap N^{g}, N^{g}\right) C_{g^{-1}}(x)=\rho^{*}\left(N \cap N^{g}, N\right)(x) \text { for all } g \in G .
$$

Here $\rho^{*}\left(H_{1}, H_{2}\right)$ is the map $\pi^{*}\left(B H_{2}\right) \rightarrow \pi^{*}\left(B H_{1}\right)$ and $C_{g^{-1}}$ is the conjugation isomorphism $\pi^{*}(B N) \rightarrow \pi^{*}\left(B N^{g}\right)$. Let $\left(\pi^{*}(B N)\right)^{s}$ denote these stable elements of $\pi^{*}(B N)$. From Feshbach [7, Theorem 2.2], we have

$$
\pi^{*}(B G) \stackrel{\rho(N, G)}{\cong}\left(\pi^{*}(B N)\right)^{s} .
$$


Applying (4.8.2) to the situation where $G=S O(3)$ and $N=O(2)$, we have to understand those $g \in G$ which give nontrivial stability conditions (4.8.1). Recall we have the following list of conjugacy classes of proper closed subgroups of $S O(3)$ and $O(2)$ from [6]:

$$
\begin{aligned}
O(2): & \left(T_{n}\right) N_{O(2)} T_{n}=O(2) \\
& \left(D_{n}\right) N_{O(2)} D_{n}=D_{2 n}, \quad n \geq 1 \\
& \left(S^{1}\right) N_{O(2)} S^{1}=O(2) \\
S O(3): \quad & \left(T_{n}\right) N_{S O(3)} T_{n}=O(2) \\
& \left(D_{n}\right) N_{S O(3)} D_{n}=D_{2 n}, n \geq 3 \\
& \left(D_{2}\right) N_{S O(3)} D_{2}=S_{4} \\
& \left(A_{4}\right) N_{S O(3)} A_{4}=S_{4} \\
& \left(S_{4}\right) N_{S O(3)} S_{4}=S_{4} \\
& \left(A_{5}\right) N_{S O(3)} A_{5}=A_{5} \\
& \left(S^{1}\right) N_{S O(3)} S^{1}=O(2) \\
& (O(2)) N_{S O(3)} O(2)=O(2)
\end{aligned}
$$

Notice that closed subgroups in $O(2)$ of order greater than two which are conjugate in $S O(3)$ are in fact conjugate in $O(2)$. Now (4.8.1) is equivalent to

$$
C_{g^{-1}} \rho^{*}\left(N \cap N^{g}, N\right)(x)=\rho^{*}\left(N \cap N^{g^{-1}}, N\right)(x) .
$$

Clearly, $O(2) \cap O(2)^{g}$ is conjugate to $O(2) \cap O(2)^{g^{-1}}$ in $S O(3)$. Using the fact that $S O(3)=S U(2) / \pm I$, it is easy to check that $O(2) \cap O(2)^{g}$ is not a conjugate of $T_{2}$ in $O(2)$ for all $g \in S O(3)$. Therefore, $O(2) \cap O(2)^{g}$ and $O(2) \cap O(2)^{g^{-1}}$ are indeed conjugate in $O(2)$. Let $\left(O(2) \cap O(2)^{g}\right)^{\alpha}=O(2) \cap O(2)^{g^{-1}}$ for some $\alpha \in O(2)$. Of course, we have

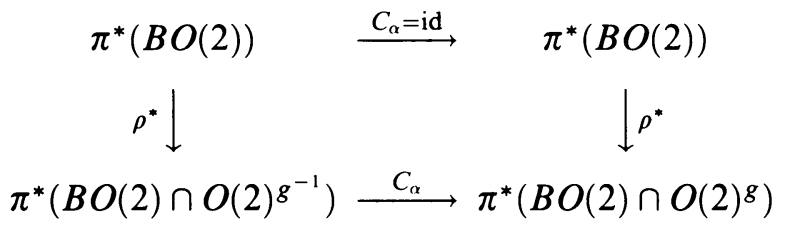

Combining this with (4.8.3), it suffices to consider the elements $x \in \pi^{*}(B O(2))$ such that

$$
\rho^{*}\left(O(2) \cap O(2)^{g}, O(2)\right)(x)=C_{g^{\prime}} \rho^{*}\left(O(2) \cap O(2)^{g}, O(2)\right)(x),
$$

where $g^{\prime}$ normalizes $O(2) \cap O(2)^{g}$ with $g \in S O(3)$. Notice that we only have to consider one representative from each conjugacy class. Clearly (4.8.4) will hold if $g^{\prime} \in O(2)$ or if $\left|O(2) \cap O(2)^{g}\right| \leq 2$. Hence we want to consider those closed subgroups of $O(2)$ with order at least three which has normalizers not contained in $O(2)$. From the given list, we see that the only possibility is $D_{2}$.

Since $D_{2}$ is abelian, $S_{4} / D_{2}=S_{3}$ acts on $D_{2}$ by conjugation. It is easy to check that in fact $S_{3}$ gives all the automorphisms of $D_{2}$. It remains to check $O(2) \cap O(2)^{g}=D_{2}$ for $g \in S_{4}-D_{4}$ since the normalizers of $D_{2}$ in $O(2)$ is $D_{4}$. So suppose $D_{2} \subset K \subset O(2) \cap O(2)^{g}$. This implies that $K^{g^{-1}} \subset O(2)$. As before, 
this implies $K^{g^{-1}}$ is conjugate to $K$ in $O(2)$. This means the normalizer of $K$ in $S O(3)$ is not contained in $O(2)$. There is only one such conjugacy class, namely $\left(D_{2}\right)$. In summary, we have

\section{Proposition 4.8.}

$$
\begin{array}{r}
\pi^{*}(B S O(3))=\left\{x \in \pi^{*}(B O(2)) \mid \rho^{*}\left(D_{2}, O(2)\right)(x)\right. \text { is invariant } \\
\text { under the action of } \left.\operatorname{Aut}\left(D_{2}\right)\right\} .
\end{array}
$$

We can interpret the proposition in terms of spectra. That is, $D B S O(3)_{+} \hat{2}$ is the summand of $D B O(2)+\hat{2}$ whose image in $D B D_{2+\hat{2}}$ is invariant under the action of $\operatorname{Aut}\left(D_{2}\right)$. An obvious approach to calculate $D B S O(3)_{+} \hat{2}$ is to split $B D_{2+\hat{2}}$ and $B O(2)^{\mathrm{Ad} O(2)} \hat{2}$ in a useful manner that will allow us to see the action of $\operatorname{Aut}\left(D_{2}\right)$. Recall the following result of Mitchell and Priddy.

Proposition 4.9 [11, Theorem C]. $B O(2)_{+} \hat{2}=B S O(3)_{+} \hat{2} \vee F$, where $B Z_{2+\hat{2}}$ is a summand of $F$.

Using this, we are able to prove

Prop ssition 4.10. $B O(2)^{\mathrm{Ad} O(2)} \hat{2}=B S O(3)^{\mathrm{Ad} S O(3)} \hat{2} \vee F$, where $F \rightarrow B O(2)+\hat{2}$ $\stackrel{j}{\longrightarrow} B O(2)^{\mathrm{Ad} O(2)} \hat{2}$ and $B S O(3)^{\mathrm{Ad} S O(3)} \hat{2} \stackrel{\mathrm{tr}}{\longrightarrow} B O(2)^{\mathrm{Ad} O(2)} \hat{2}$ are the maps that give the equivalence. Note $j$ is induced from $\{e\} \hookrightarrow \operatorname{Ad} O(2)$.

Proof. It suffices to prove isomorphism in mod 2 cohomology. Recall we have

$$
H^{*}\left(B S O(3), Z_{2}\right)=Z_{2}\left[\bar{\omega}_{2}, \bar{\omega}_{3}\right], \quad H^{*}\left(B O(2), Z_{2}\right)=Z_{2}\left[\omega_{1}, \omega_{2}\right],
$$

where $\pi^{*}\left(\bar{\omega}_{2}\right)=\omega_{2}+\omega_{1}^{2}, \pi^{*}\left(\bar{\omega}_{3}\right)=\omega_{1} \omega_{2}$, and $\pi: B O(2) \rightarrow B S O(3)$. Via the Thom isomorphism,

$$
H^{*}\left(B O(2)^{\operatorname{Ad} O(2)}, Z_{2}\right) \stackrel{j^{*}}{\longrightarrow} H^{*}\left(B O(2), Z_{2}\right)
$$

can be identified with

$$
\bar{H}^{*-1}\left(B O(2), Z_{2}\right) \stackrel{\times \omega_{1}}{\longrightarrow} H^{*}\left(B O(2), Z_{2}\right)
$$

since the Euler class of $\operatorname{Ad} O(2)$ is $\omega_{1}$.

We want to show that

$$
\bar{H}^{*}\left(B O(2)^{\mathrm{Ad} O(2)}, Z_{2}\right) \rightarrow \bar{H}^{*}\left(B S O(3)^{\mathrm{Ad} S O(3)}, Z_{2}\right) \oplus \bar{H}^{*}\left(F, Z_{2}\right)
$$

is an isomorphism. Since $B O(2)+\hat{2}=B S O(3)+\hat{2} \vee F$, the kernel of

$$
H^{*}\left(B O(2), Z_{2}\right) \rightarrow H^{*}\left(F, Z_{2}\right)
$$

can be identified via $H^{*}\left(B S O(3), Z_{2}\right)$ as

$$
\sum a_{i j}\left(\omega_{2} \omega_{1}\right)^{i}\left(\omega_{2}+\omega_{1}^{2}\right)^{j}
$$

Hence the kernel of

$$
H^{*}\left(B O(2)^{\text {Ad } O(2)}, Z_{2}\right) \stackrel{j^{*}}{\longrightarrow} H^{*}\left(B O(2), Z_{2}\right) \rightarrow H^{*}\left(F, Z_{2}\right)
$$

can be written as

$$
K=\sum a_{i j}^{\prime} \omega_{2}\left(\omega_{2} \omega_{1}\right)^{i}\left(\omega_{2}+\omega_{1}^{2}\right)^{j}
$$


We claim $\operatorname{tr}^{*}(K) \neq 0$ of $K \neq 0$, where $\operatorname{tr}: B S O(3)^{\operatorname{Ad} B O(3)} \hat{2} \rightarrow B O(2)^{\operatorname{Ad} O(2)} \widehat{2}$. By Thom isomorphism, $\operatorname{tr}^{*}$ corresponds to

$$
t_{!}: H^{*}\left(B O(2), Z_{2}\right) \rightarrow H^{*-2}\left(B S O(3), Z_{2}\right) .
$$

Since $S O(3) / O(2)=R P^{2}$ and $i^{*}\left(\omega_{1}^{2}\right)=[F]$, the fundamental class of $R P^{2}$ where $R P^{2} \stackrel{i}{\rightarrow} B O(2)$, we have $t_{!}\left(\omega_{1}^{2}\right)=1$ (cf. [13, Proposition 2.2]). By naturality, we have $t_{!}\left(\omega_{1}^{2}+\omega_{2}\right)=t_{!}\left(\pi^{*}\left(\bar{\omega}_{2}\right)\right)=t_{!}(1) \bar{\omega}_{2}=0$. Therefore $t_{!}\left(\omega_{2}\right)=$ 1. Hence,

$$
\begin{aligned}
t_{!}(K) & =t_{!}\left(\sum a_{i j}^{\prime} \omega_{2}\left(\omega_{2} \omega_{1}\right)^{i}\left(\omega_{2}+\omega_{1}^{2}\right)^{j}\right) \\
& =t_{!}\left(\sum a_{i j}^{\prime} \omega_{2} \pi^{*}\left(\bar{\omega}_{3}\right)^{i} \pi^{*}\left(\bar{\omega}_{2}\right)^{j}\right) \\
& =\sum a_{i j}^{\prime} \bar{\omega}_{3}^{i} \bar{\omega}_{2}^{j} \\
& \neq 0 \quad \text { unless } a_{i j}^{\prime}=0 \text { for all } i, j .
\end{aligned}
$$

To show surjectivity, we just check the Poincaré series of the target and the source are equal. Therefore we are done.

Theorem 4.11 [11, Theorem A]. $B D_{2+2}=B A_{4+2} \vee F \vee F$, where the first summand $F$ can be mapped into the second summand $F$ by the automorphism $\tau \sigma$ in $\operatorname{Aut}\left(D_{2}\right)=G L_{2}\left(Z_{2}\right)$; here $\tau=\left(\begin{array}{ll}0 & 1 \\ 1 & 0\end{array}\right)$ and $\sigma=\left(\begin{array}{ll}0 & 1 \\ 1 & 1\end{array}\right)$.

Remark. $F$ coincides with the summand $F$ in the splitting of $B O(2)+\hat{2}$. We discuss some details of the splitting so that we can understand the action of $\operatorname{Aut}\left(D_{2}\right)$ with respect to this decomposition. First of all, the summands $F_{1} \vee$ $F_{2}$ (the subscripts are for distinction) come from splitting using idempotents in $Z_{2}\left[G L_{2}\left(Z_{2}\right)\right]$. Specifically, let $\pi_{2}=\sigma+\sigma^{2}, \rho_{1}=(1+\tau \sigma)(1+\tau)$, and $\rho_{2}=(1+\tau)(1+\tau \sigma)$. We have $\pi_{2}=\rho_{1}+\rho_{2}, \rho_{1} \rho_{2}=\rho_{2} \rho_{1}=0$. Then $H^{*}\left(F_{i}, Z_{2}\right)=\operatorname{Im} \rho_{i}^{*} \subset H^{*}\left(B D_{2}, Z_{2}\right)$ for $i=1,2$. We remark that elements are acting from the right on cohomology.

We claim that $\operatorname{Aut}\left(D_{2}\right)$ acts as automorphism $G L_{2}\left(Z_{2}\right)$ on $F_{1} \vee F_{2}$. To see the reason, we already know $\rho_{1}, \rho_{2}$ are projection onto the first or second coordinate while $\tau \sigma$ switch the coordinates. Consider $\tau$. We have $\rho_{1} \tau=\rho_{1}$. This implies that the action of $\tau$ on $F_{1}$ maps it to itself. $\rho_{2} \tau \rho_{1}=\rho_{2}(\sigma \tau)$, and $\rho_{2} \tau \rho_{2}=\rho_{2}$ implies that $\tau$ maps $F_{2}$ to $F_{1} \vee F_{2}$ by diagonal. Similarly, we get all the other elements of $G L_{2}\left(Z_{2}\right)$.

As for $B A_{4+2}$, this comes from the transfer associated to $D_{2} \hookrightarrow A_{4}$. Therefore it is obvious that the action of $\operatorname{Aut}\left(D_{2}\right)$ on $B A_{4+2}$ is trivial.

Lemma 4.12. Let $K$ be a compact Lie group and $\sigma \in \operatorname{Aut}(K)$. Then the action of $\sigma$ on $\left(S^{0}\right)^{K}$ is given by $B W_{H}$ Ad $W_{H} \rightarrow B W_{\sigma H}^{\mathrm{Ad} W_{\sigma H}}$.

Proof. To avoid confusion, write $\sigma: K_{1} \rightarrow K_{2}$. As a $K_{1}$-space, $K_{2} / N H=$ $K_{1} / \sigma^{-1}(N H)=K_{1} / N\left(\sigma^{-1} H\right)$. By the naturality of tom Dieck's splitting with respect to the conjugation isomorphism, we have the result.

By the lemma, it is easy to see that $\operatorname{Aut}\left(D_{2}\right)=S_{3}$ acts on $D B D_{2+}$ by conjugation on the summand $B D_{2+}$, permuting $B Z_{2+} \vee B Z_{2+} \vee B Z_{2+}$, and leaves $S^{0}$ alone. 
Theorem 4.13.

Proof. Consider the split cofiber sequence

$$
\begin{aligned}
D B S O(3)_{+} \simeq & S_{2}^{0} \hat{2} \vee B S O(3)^{\mathrm{Ad} S O(3)} \hat{2} \\
& \vee \prod_{1}^{\infty} B O(2)^{\mathrm{Ad} O(2)} \hat{2} \vee \prod_{1}^{\infty} B Z_{2+\hat{2}} .
\end{aligned}
$$

$$
D B O(2)_{+} \hat{2} \stackrel{D[B r]}{\longrightarrow} B B O(2)_{+} \hat{2} \rightarrow B O(2)^{\mathrm{Ad} O(2)} \hat{2} \vee B Z_{2+\hat{2}} .
$$

We may rewrite $(4.13 .1)$ as

$$
D B W_{+} \hat{2} \vee \mathcal{J} \stackrel{D[B r]}{\longrightarrow} D B O(2)_{+} \hat{2} \rightarrow B O(2)^{\mathrm{Ad} O(2)} \hat{2} \vee B Z_{2+\hat{2}},
$$

where $W=Z_{2}$ is the Weyl group of $O(2)$ and $\mathscr{J}=\prod_{1}^{\infty} B O(2)^{\operatorname{Ad} O(2)} \hat{2} \vee$ $\prod_{1}^{\infty} B Z_{2+\hat{2}}$. Let $\Theta$ be the composite

$$
\mathscr{J} \hookrightarrow B D W_{+2} \vee \mathcal{J} \stackrel{D[B r]}{\longrightarrow} D B O(2)_{+2} \stackrel{D[B j]}{\longrightarrow} D B D_{2+\hat{2}} .
$$

By the commutative diagram

$$
\begin{aligned}
& D B O(2)_{+} \hat{2} \stackrel{D[B r]}{\longrightarrow} D B O(2)+\hat{2} \\
& D[B j] \downarrow \downarrow D[B j] \\
& D B D_{1+\hat{2}} \stackrel{D[B r]}{\longrightarrow} D B D_{2+\hat{2}}
\end{aligned}
$$

we see that $\Theta$ is a map between $\mathcal{J}$ and $B Z_{2+\hat{2}}$ if we identify $D B D_{1+\hat{2}}$ as $S^{0} \hat{2} \vee B Z_{2+} \hat{2}$. Consider the map

$$
\mathscr{J} \stackrel{\text { id } \vee \Theta}{\longrightarrow} \mathscr{J} \vee B W_{+} \hat{2} \hookrightarrow \mathscr{J} \vee D B W_{+2} \stackrel{D[B r]}{\longrightarrow} D B O(2)_{+} \widehat{2} \stackrel{D[B j]}{\longrightarrow} D B D_{2+} \hat{2} \text {. }
$$

By construction, this is the zero map. Thus $\mathcal{J}$ is a summand of $D B O(2)+\hat{2}$, via id $\vee \Theta$, whose image in $D B D_{2+\hat{2}}$ is invariant under the action of $\operatorname{Aut}\left(D_{2}\right)$.

Now the summand $B Z_{2+\hat{2}}$ maps to $D B D_{2+\hat{2}}$ under $D[B j]$ according to the description in Lemma 4.7, and $B W_{+} \hat{2}$ maps to the summand in $D B D_{2+} \hat{2}$ corresponding to $\left(T_{2}\right)$ by the identity. From the comment made after Lemma 4.12 , it is clear that we have to analyze the map $B Z_{2+\widehat{2}} \stackrel{B i}{\longrightarrow} B D_{2+\widehat{2}}$ and understand how it relates to the bundle transfer $\operatorname{tr}: B O(2)^{\operatorname{Ad} O(2)} \hat{2} \rightarrow B D_{2+\hat{2}}$. We use the splittings of $B O(2)^{\mathrm{Ad}} O(2) \hat{2}$ and $B D_{2+} \hat{2}$ from Propositions 4.10 and 4.11 to analyze the bundle transfer map $\operatorname{tr}$.

In [11, Theorem $C$ ], it was shown that the summand $F$ of $B O(2)+\hat{2}$ factors through $B D_{2+\hat{2}} \stackrel{B i}{\longrightarrow} B O(2)_{+} \hat{2}$, where $F$ maps to the summand $F_{1}$. The composite

$$
B O(2)_{+} \hat{p} \rightarrow B O(2)^{\mathrm{Ad} O(2)} \hat{2} \stackrel{\operatorname{tr}}{\longrightarrow} B D_{2+\hat{2}}
$$

is equal to the ordinary transfer associated to $D_{2} \hookrightarrow O(2)$. Therefore we have the following diagram:

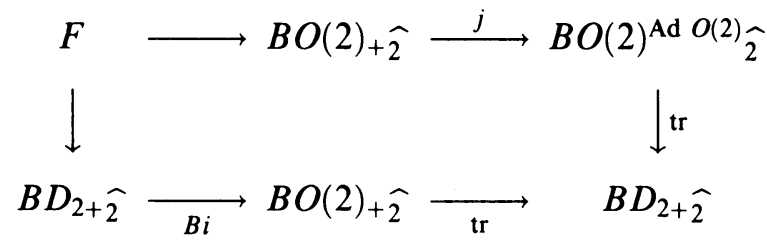


By the double coset theorem, $B D_{2+\hat{2}} \underset{B i}{\longrightarrow} B O(2)_{+} \hat{2} \underset{\operatorname{tr}}{\longrightarrow} B D_{2+\hat{2}}$ is the same as $1+C_{\tau \sigma}$. Therefore $F$ of $B O(2)^{\mathrm{Ad} O(2)} \hat{2}$ maps diagonally into $F_{1} \vee F_{2}$ of $B D_{2+\hat{2}}$. Clearly, the summand $B S O(3)^{\mathrm{Ad} S O(3)} \hat{2}$ of $B O(2)^{\mathrm{Ad} O(2)} \hat{2}$ maps to the summand $B A_{4+\hat{2}}$ of $B D_{2+\hat{2}}$ by bundle transfer.

Since we remarked that $B Z_{2+\hat{2}}$ is a summand of $F$, it is clear then from the discussion following Proposition 4.11 that the summand $F \vee B Z_{2+} \widehat{2} \vee B W_{+} \hat{2}$ of $\mathrm{DBO}(2)+\hat{2}$ cannot contribute to a stable summand of $\mathrm{DBSO}(3)+\hat{2}$. Therefore we have

$$
\mathrm{DBSO}(3)_{+} \simeq \mathrm{S}^{0} \hat{\mathrm{z}} \vee \mathscr{J} \vee \mathrm{BSO}(3)^{\mathrm{Ad} S O(3)} \hat{2} .
$$

Recall Miller and Wilkerson proved

Theorem 4.14 [12, Theorem B]. $D B S^{3}+\hat{2} \simeq B S^{3 A d} B S^{3} \hat{2} \vee D B S O(3)+\hat{2}$.

Theorem 4.15 [12, Theorem B]. $D B N_{2+} \widehat{2} \simeq B N_{2}^{\mathrm{Ad} B N_{2}} \hat{2} \vee D B O(2)_{+} \hat{2}$, where $N_{2}$ denotes the normalizer of the maximal torus in $S^{3}$.

Remark. We can recover Theorem 4.15 from Proposition 4.3 for there is no nontrivial 2-group in $N_{2}$ whose intersection with the normal subgroup $\{ \pm I \in$ $\left.S^{3}\right\}$ is trivial.

From Theorem 4.13 and Proposition 4.6, we get

Corollary 4.16.

$$
\begin{aligned}
D B S^{3}+\hat{2} \simeq & S^{0} \hat{2} \vee B S^{3 \mathrm{Ad} B S^{3}} \hat{2} \vee B S O(3)^{\mathrm{Ad} S O(3)} \hat{2} \\
& \vee \prod_{1}^{\infty} B O(2)^{\mathrm{Ad} O(2)} \hat{2} \vee \prod_{1}^{\infty} B Z_{2+\hat{2}}
\end{aligned}
$$

Corollary 4.17.

$$
\begin{aligned}
D B N_{2+\hat{2}}= & S_{2}^{0} \hat{2} \vee B Z_{2+\hat{2}} \vee B N_{2}^{\mathrm{Ad} B N_{2}} \hat{2} \\
& \vee \prod_{1}^{\infty} B O(2)^{\mathrm{Ad} O(2)} \hat{2} \vee \prod_{1}^{\infty} B Z_{2+\hat{2}} .
\end{aligned}
$$

\section{REFERENCES}

1. J. F. Adams, Stable homotopy and generalized homology, Univ. of Chicago Press, Chicago, 1974.

2. A. Bousfield and D. Kan, Homotopy limits, completions, and localizations, Lecture Notes in Math., vol. 304, Springer-Verlag, New York, 1972.

3. G. Bredon, Introduction to compact transformation groups, Academic Press, New York, 1972.

4. G. Carlsson, Equivariant stable homotopy and Segal's Burnside ring conjecture, Ann. of Math. 120 (1984), 189-224.

5. T. tom Dieck, Orbittypen und äquivariante Homologie. II, Arch. Math. 26 (1975), 650-662.

6. __ Transformation groups and representation theory, Lecture Notes in Math., vol. 766, Springer-Verlag, New York, 1979.

7. M. Feshbach, The Segal conjecture for compact Lie group, Topology 26 (1987), 1-20.

8. L. Lewis, J. May and J. McClure, Classifying G-spaces and the Segal conjecture, Canad. Math. Soc. Conf. Proc., Vol. 2, Part 2, Amer. Math. Soc., Providence, R. I., 1982, pp. 165-179. 
9. L. Lewis, J. May and M. Steinberger, Equivariant stable homotopy theory, Lecture Notes in Math., vol. 1213, Springer-Verlag, New York, 1986.

10. B. Mann, E. Miller, and H. Miller, $S^{1}$-equivariant function spaces and characteristic classes, Trans. Amer. Math. Soc. 295 (1986), 233-256.

11. S. Mitchell and S. Priddy, Symmetric product spectra and splittings of classifying spaces, Amer. J. Math. 106 (1984), 219-232.

12. H. Miller and C. Wilkerson, On the Segal conjecture for periodic groups, Contemporary Math., Amer. Math. Soc., Providence, R. I., 1983, pp. 233-246.

13. G. Nishida, On the $S^{1}$-Segal conjecture, Publ. Res. Inst. Math. Sci. 19 (1983), 1153-1162.

14. D. Ravenel, The Segal conjecture for cyclic groups and its consequences, Amer. J. Math. 106 (1984), 415-446.

Department of Mathematics, Princeton University, Princeton, New Jersey 08544

Current address: Department of Mathematics, Northwestern University, 2033 Sheridan Road, Evanston, Illinois 60208

E-mail address: clee@hilbert.math.nwu.edu 